\title{
Characterization of carbonyl disinfection by-products during ozonation, chlorination and chloramination of dissolved organic matters
}

Xueke Liu ${ }^{1,2}$, Ruirui Liu ${ }^{1}$, Bao Zhu ${ }^{1}$, Ting Ruan ${ }^{1,2} *$ and Guibin Jiang ${ }^{1,2}$

${ }^{1}$ State Key Laboratory of Environmental Chemistry and Ecotoxicology, Research Center for Eco-Environmental Sciences, Chinese Academy of Sciences, Beijing 100085, China

${ }^{2}$ University of Chinese Academy of Sciences, Beijing 100049, China

*Corresponding author

Dr. Ting Ruan

Research Center for Eco-Environmental Sciences,

Chinese Academy of Sciences

Tel: 8610-6284-9334

Fax: 8610-6284-9179

E-mail: tingruan@rcees.ac.cn

Number of pages: 35

Number of Tables: 8

Number of Figures: 12 


\section{Contents}

CONTENT1. Free chlorine measurement

CONTENT2. Data analysis of FT-ICR mass spectrum

CONTENT3. LC-MS parameters for $\mathrm{MS}^{2}$ spectrum

CONTENT4. Optimization of the derivatization method

CONTENT5. Quality assurance of the derivation method

Table S1. Detailed information on compositions of DOM-control samples (disinfectants-before) and experimental samples (disinfectants-after), disinfection types, and reaction duration time.

Table S2. Mass list of known DOM homologous series for internal calibration of FTICR mass spectra in the ESI' mode.

Table S3. Mass list of known DOM homologous series for internal calibration of FTICR mass spectra in the $\mathrm{ESI}^{+}$mode.

Table S4. An in-house reference mass list of carbonyl compounds for internal calibration of FT-ICR mass spectra in the $\mathrm{ESI}^{+}$mode.

Table S5. Magnitude-weighted average values of molecular characteristics of carbonyl compounds generated at the varied derivatization reaction temperatures.

Table S6. Magnitude-weighted average values of molecular characteristics of $\mathrm{CHO}$ carbonyl compounds before and after disinfection (ozonation, chlorination and chloramination).

Table S7. A summary of the halogenated carbonyl DBPs reported in literature (with only one possible isomer shown in the table).

Table S8. Molecular structures of known DBPs mentioned in main text.

Figure S1. Optimization of derivatization conditions for carbonyl compounds in the SRFA reference standard, including temperature (a), amounts of GRP reagent (b), incubation time (c) and acidity (d). 
Figure S2. Venn diagram on number of carbonyl compounds identified in the three parallel SRFA aqueous samples.

Figure S3. An illustration on derivatization reaction of a representative carbonyl compound $\left(\mathrm{C}_{10} \mathrm{H}_{10} \mathrm{O}_{6}\right)$ in the SRFA reference standard with $\mathrm{D}_{0} / \mathrm{D}_{5^{-}} \mathrm{GRP}(\mathrm{a})$, and $\mathrm{MS}^{2}$ spectra of the corresponding $\mathrm{D}_{0} / \mathrm{D}_{5}$-GRP derivatives $\left(\mathrm{b},\left[\mathrm{C}_{17} \mathrm{H}_{18} \mathrm{~N}_{3} \mathrm{O}_{6}\right]^{+}\right.$and $\left.\left[\mathrm{C}_{17} \mathrm{H}_{13} \mathrm{D}_{5} \mathrm{~N}_{3} \mathrm{O}_{6}\right]^{+}\right)$.

Figure S4. Proposed average structural model of the SRFA reference standard (a), proposed structure of a lignin molecule $\left(\mathrm{C}_{24} \mathrm{H}_{28} \mathrm{O}_{11}, \mathrm{~b}\right)$, and several depolymerization products of lignin (c). The moieties of hydroxyphenyl alkyl ketones were circled by red dotted line in the structures of fulvic acid and lignin.

Figure S5. Relative abundance and formation of carbonyl molecules in the SRFA solution after ozonation $(\mathrm{a}, \mathrm{d})$, chlorination $(\mathrm{b}, \mathrm{e})$, and chloramination $(\mathrm{c}, \mathrm{f})$ by van Krevelen plots. In d-f, red points referred to the newly produced carbonyl compounds (DBPs), and black triangles were original carbonyl compounds existed and remained in the solution.

Figure S6. Molecular characteristic alteration of carbonyl compounds in HOCs region $(0.67 \leqslant \mathrm{O} / \mathrm{C} \leqslant 1.2$ and $0.6<\mathrm{H} / \mathrm{C} \leqslant 1.5)$ after ozonation. The number of produced (red), remained (black) and disappeared (green) carbonyl compounds shown by oxygen number (a); DBE composition of the produced carbonyl compounds (carbonyl DBPs) in $\mathrm{O}_{4-10}$ species (b); KMD plots of representative carbonyl DBPs with proposed structures (c).

Figure S7. Distribution of carbonyl compound numbers shown by oxygen number, and DBE distribution shown by oxygen number after ozonation $(a, b)$, chlorination $(c, d)$ and chloramination (e, f).

Figure S8. KMD plots of Cl-carbonyl-DBP homologous $\left(\mathrm{O}_{3}, \mathrm{DBE}=2-3 ; \mathrm{O}_{4}, \mathrm{DBE}=\right.$ 4; $\mathrm{O}_{5}, \mathrm{DBE}=5$ ) in chloramine-treated SRFA solution.

Figure S9. $\mathrm{MS}^{2}$ spectra of $\mathrm{D}_{0} / \mathrm{D}_{5}-\mathrm{GRP}$ derivatives of $\mathrm{C}_{7} \mathrm{H}_{5} \mathrm{O}_{5} \mathrm{Cl}$ (a) and $\mathrm{C}_{9} \mathrm{H}_{9} \mathrm{O}_{5} \mathrm{Cl}$ (b) with $\mathrm{DBE}=5$ in $\mathrm{O}_{5}$ species.

Figure S10. $\mathrm{MS}^{2}$ spectra of $\mathrm{D}_{0} / \mathrm{D}_{5}$-GRP derivatives of $\mathrm{C}_{6} \mathrm{H}_{4} \mathrm{O}_{4} \mathrm{Cl}_{2}\left(\mathrm{a}, \mathrm{DBE}=4\right.$ in $\mathrm{O}_{4}$ species), $\mathrm{C}_{5} \mathrm{H}_{5} \mathrm{O}_{3} \mathrm{Cl}$ (b, DBE $=3$ in $\mathrm{O}_{3}$ species), $\mathrm{C}_{5} \mathrm{H}_{6} \mathrm{O}_{3} \mathrm{Cl}_{2}$ (c, DBE $=2$ in $\mathrm{O}_{3}$ species), and $\mathrm{C}_{4} \mathrm{H}_{3} \mathrm{O}_{3} \mathrm{Cl}\left(\mathrm{d}, \mathrm{DBE}=3\right.$ in $\mathrm{O}_{3}$ species).

Figure S11. Molecular characteristics of Br-carbonyl-DBPs after chlorination and chloramination. The number of Br-carbonyl-DBPs induced by chlorine (green) and chloramine (blue) shown by oxygen number (a); DBE composition of $\mathrm{O}_{3}$ species (b); 
KMD plots of the Br-carbonyl-DBP homologous $\left(\mathrm{O}_{3}, \mathrm{DBE}=3\right)$ with representative proposed structures (c); $\mathrm{MS}^{2}$ spectra of $\mathrm{D}_{0} / \mathrm{D}_{5}$-GRP derivatives of $\mathrm{C}_{4} \mathrm{H}_{3} \mathrm{O}_{3} \mathrm{Br}(\mathrm{d})$.

Figure S12. Molecular characteristics of I-carbonyl-DBPs after chloramination and ozonation. The number of I-carbonyl-DBPs induced by chloramine (blue) and ozone (red) shown by oxygen number (a); DBE composition of $\mathrm{O}_{3-5}$ class species (b); KMD plots of the I-carbonyl-DBP homologous $\left(\mathrm{O}_{3}, \mathrm{DBE}=2-3 ; \mathrm{O}_{4}, \mathrm{DBE}=4 ; \mathrm{O}_{5}, \mathrm{DBE}=\right.$ 5) with representative proposed structures (c); $\mathrm{MS}^{2}$ spectra of $\mathrm{D}_{0} / \mathrm{D}_{5}-\mathrm{GRP}$ derivatives of $\mathrm{C}_{4} \mathrm{H}_{3} \mathrm{O}_{3} \mathrm{I}(\mathrm{d})$. 
1 CONTENT1. Free chlorine measurement

2

3 Chlorine stock solution was standardized by using the N,N-diethyl-p-phenylenediamine (DPD) assay (China National Standard GB/T 5750.11-2006). Briefly, chlorine stock solution was diluted, $10 \mathrm{~mL}$ of which was then mixed with $0.5 \mathrm{~mL}$ of $1 \mathrm{~g} / \mathrm{L}$ DPD solution (including $0.2 \% \mathrm{H}_{2} \mathrm{SO}_{4}$ and $0.2 \mathrm{~g} / \mathrm{L} \mathrm{Na}$-EDTA) and $0.5 \mathrm{~mL}$ of $0.5 \mathrm{M}$ phosphate buffer solution ( $\mathrm{pH}=6.5$, containing $0.8 \mathrm{~g} / \mathrm{L} \mathrm{Na} 2$-EDTA). Absorbance of the mixture was measured by UV-Vis spectrophotometer at $515 \mathrm{~nm}$ for quantitative analysis. $\mathrm{KMnO}_{4}$ solution $(0.06-4.51 \mu \mathrm{M})$ was separately used as oxidant alternative in the assay to obtain the linear curve for free chlorine quantification in the range of $0.14-$ 11.27 $\mu \mathrm{M}$. The chlorine stock solution concentration was presented in the form of the measured free chlorine concentration. Measurement of monochloramine stock solution concentration followed the same procedure. No significant signal was observed during the spectrophotometer analysis, indicating little free chlorine interferent in the mixture. Then, $0.1 \mathrm{~mL}$ potassium iodide solution $(1.0 \mathrm{~g} / \mathrm{L})$ was added to the mixture, and absorbance of the mixture was measured again. The measure data were used to quantify monochloramine concentrations. The assay was also used to measure residue chlorine after disinfection by mixing $0.25 \mathrm{~mL}$ of chlorine-treated SRFA solution with $0.5 \mathrm{~mL}$ of $1 \mathrm{~g} / \mathrm{L}$ DPD solution, $0.5 \mathrm{~mL}$ of $0.5 \mathrm{M}$ phosphate buffer solution and $9.75 \mathrm{~mL}$ of ultrapure water. After 5 days of chlorination, the residual chlorine concentrations of SRFA solution were $0.130 \mathrm{mM}, 0.125 \mathrm{mM}$ (adding $\mathrm{Br}^{-}$) and $0.114 \mathrm{mM}$ (adding $\mathrm{I}^{-}$), accounting for $19 \%, 19 \%$ and $17 \%$ of initially-dosed chlorine concentrations, respectively. 
CONTENT2. Data analysis of FT-ICR mass spectrum

The van Krevelen diagram was commonly used to visualize chemical characteristics by construction of $\mathrm{O} / \mathrm{C}$ and $\mathrm{H} / \mathrm{C}$ ratios in molecular formulas. Compounds were classified into seven groups, including lipids-like $(0 \leq \mathrm{O} / \mathrm{C} \leq 0.3,1.5<\mathrm{H} / \mathrm{C} \leq 2.0)$, aliphatic/peptides-like $(0.3<\mathrm{O} / \mathrm{C} \leq 0.67,1.5<\mathrm{H} / \mathrm{C} \leq 2.2)$, carbohydrates-like $(0.67<$ $\mathrm{O} / \mathrm{C} \leq 1.2,1.5<\mathrm{H} / \mathrm{C} \leq 2.5$ ), lignin or carboxylic-rich alicyclic molecules structures (lignin/CRAMs-like structures, $0.1 \leq \mathrm{O} / \mathrm{C}<0.67,0.67<\mathrm{H} / \mathrm{C} \leq 1.5$ ), unsaturated hydrocarbons $(0 \leq \mathrm{O} / \mathrm{C}<0.1,0.67<\mathrm{H} / \mathrm{C} \leq 1.5)$, aromatic structures $(0 \leq \mathrm{O} / \mathrm{C}<0.67$, $0.3 \leq \mathrm{H} / \mathrm{C} \leq 0.67)$, and highly oxygenated compounds $(0.67 \leq \mathrm{O} / \mathrm{C} \leq 1.2,0.6<\mathrm{H} / \mathrm{C} \leq$ 1.5). The same Kendrick mass defect (KMD) values represent homologous compounds with extension of $\mathrm{CH}_{2}$. Kendrick mass (KM) and $\mathrm{KMD}$ were calculated by equation 1 and 2. DBE was calculated by equation 3 , where $\mathrm{c}, \mathrm{h}$, and $\mathrm{x}$ refer to the number of carbon, hydrogen and halogen in formulas, respectively. Magnitude-weighted averages of $\mathrm{O} / \mathrm{C}, \mathrm{H} / \mathrm{C}, \mathrm{DBE}, \mathrm{DBE} / \mathrm{C}$ and molecular weight (MW), expressed as $\mathrm{O} / \mathrm{C}_{\mathrm{w}}, \mathrm{H} / \mathrm{C}_{\mathrm{w}}$, $\mathrm{DBE}_{\mathrm{w}}, \mathrm{DBE} / \mathrm{C}_{\mathrm{w}}$ and $\mathrm{MW}_{\mathrm{w}}$, were calculated by the equation 4 , where $\mathrm{I}_{\mathrm{i}}$ and $\mathrm{A}_{\mathrm{i}}$ refer to intensity and molecular characteristics of peak i, respectively. The measured values of CHO carbonyl molecules before and after ozonation, chlorination and chloramination were shown in Table S5.

$$
\begin{aligned}
& \mathrm{KM}=\mathrm{IUPAC} \text { mass } \times(14 \div 14.01565) \\
& \mathrm{KMD}=\text { absolute }(\text { nominal } \mathrm{KM}-\mathrm{KM}) \\
& \mathrm{DBE}=\mathrm{c}+1-\frac{\mathrm{h}}{2}-\frac{\mathrm{x}}{2} \\
& \mathrm{~A}_{w}=\sum\left(\mathrm{I}_{\mathrm{i}} \times \mathrm{A}_{\mathrm{i}}\right) / \sum \mathrm{I}_{\mathrm{i}}
\end{aligned}
$$


CONTENT3. LC-MS parameters for $\mathrm{MS}^{2}$ spectrum

$\mathrm{MS}^{2}$ spectra were mainly used for molecular structure confirmation of the identified carbonyl compounds, which were obtained from the Orbitrap Fusion mass spectrometer coupled with Ultimate 3000 ultrahigh performance liquid chromatography (UPLCOrbitrap HRMS, Thermo Fisher Scientific Inc., Waltham, MA). An ACQUITY BHE HILIC column (Waters, $1.7 \mu \mathrm{m}, 2.1 \times 100 \mathrm{~mm}$ ) was maintained at $35^{\circ} \mathrm{C}$ for analyte separation. A constant mobile phase of acetonitrile and water $(85 / 15, \mathrm{v} / \mathrm{v})$ was used, and the flow rate was $0.3 \mathrm{~mL} / \mathrm{min}$. Injection volume was $5 \mu \mathrm{L}$, and the compounds were ionized in positive electrospray ionization (ESI). Spray voltage was set as $3500 \mathrm{~V}$, and vaporizer temperature was $200{ }^{\circ} \mathrm{C}$. The pressures of sheath gas and aux gas were 35 and 15 Arb, respectively. The mass spectrometry method contained two parallel events: (1) full scan mode was selected, and orbitrap was used with the resolution of 120,000 (FWHM at $\mathrm{m} / \mathrm{z} 200$ ) and the scan range of $\mathrm{m} / \mathrm{z}=160-800$. (2) $\mathrm{MS}^{2}$ mode was selected using higher-energy collisional dissociation (HCD) mode with $25 \pm 10 \%$ of collision energy, and the $\mathrm{MS}^{1}$ precursors were isolated by quadrupole with window of $\mathrm{m} / \mathrm{z}=1$. Fragmentation ions were detected with a resolution of 30,000 (FWHM at m/z 200). 
CONTENT4. Optimization of the derivatization method

In order to obtain a maximum derivatization efficiency, conditions including reaction temperature, time, amounts of GRP reagent and acidity were optimized (Figure S1). During optimization, the Suwannee River Fulvic Acid II (SRFA, 2S101F) reference standard was used with dissolved organic matters (DOM) concentration kept at 0.083 mmol C, which was the same as that in the disinfection experiments. Firstly, the reaction temperature was optimized that $200 \mu \mathrm{L} \mathrm{DOM}(0.083 \mathrm{mmol})$ was mixed with $400 \mu \mathrm{L}$ of $2 \mathrm{mM} \mathrm{HCl}, 300 \mu \mathrm{L}$ of $5 \mathrm{mM} \mathrm{D}$-GRP and $300 \mu \mathrm{L}$ of $5 \mathrm{mM} \mathrm{D}$-GRP at different temperatures ranging from 30 to $60{ }^{\circ} \mathrm{C}$ for $10 \mathrm{~min}$. The maximum number of carbonyl compounds $(\mathrm{n}=511 \pm 20)$ was observed at $50{ }^{\circ} \mathrm{C}$. No significant difference was found among the carbonyl compounds generated at the varied temperatures in magnitudeweighted average values of each molecular characteristics (Table $\mathrm{S} 5, \mathrm{O} / \mathrm{C}_{\mathrm{w}}, \mathrm{H} / \mathrm{C}_{\mathrm{w}}$, $\mathrm{DBE}_{\mathrm{w}}, \mathrm{DBE} / \mathrm{C}_{\mathrm{w}}$ and $\mathrm{MW}_{\mathrm{w}}$ ), and $50{ }^{\circ} \mathrm{C}$ was thus selected for optimization of other conditions. Amounts of GRP reagent were optimized that $0.5-10 \mathrm{mM}$ of $\mathrm{D}_{0}$-GRP and D5-GRP were mixed with $200 \mu \mathrm{L}$ DOM $(0.083 \mathrm{mmol})$ and $400 \mu \mathrm{L}$ of $2 \mathrm{mM} \mathrm{HCl}$ at $50{ }^{\circ} \mathrm{C}$ for $10 \mathrm{~min}$. With the increase of $\mathrm{D}_{0} / \mathrm{D}_{5}$-GRP concentrations, the number of carbonyl compounds reached a plateau (Figure S1b). Thus, $5 \mathrm{mM}$ of $\mathrm{D}_{0}-\mathrm{GRP}$ and $\mathrm{D}_{5-}$ GRP was selected. Derivatization time was optimized in the range of 10 to $120 \mathrm{~min}$. Results showed that derivatization reaction was fast that 10 min was enough for efficient reaction (Figure S1c). The amounts of $\mathrm{HCl}$ were optimized ranging from 0.5 to $10 \mathrm{mM}$. The optimum derivatization efficiency can be achieved when $2 \mathrm{mM} \mathrm{HCl}$ was used (Figure S1d). Based on these results, carbonyl compounds in the SRFA 
88 reference standard were derivatized by adding $300 \mu \mathrm{L}$ of $5 \mathrm{mM} \mathrm{D} \mathrm{D}_{0}$-GRP, $300 \mu \mathrm{L}$ of 5

$89 \mathrm{mM} \mathrm{D}_{5}-\mathrm{GRP}$ and $400 \mu \mathrm{L} 2 \mathrm{mM} \mathrm{HCl}$ at $50{ }^{\circ} \mathrm{C}$ for $10 \mathrm{~min}$. 
CONTENT5. Quality assurance of the derivatization method

The Venn diagram of carbonyl compounds detected in three parallel SRFA samples was used to show reproducibility of the method (Figure S2). On average, $511 \pm 20$ carbonyl compounds were observed, in which 429 ones were ubiquitous components in each sample. Figure 1 showed that $97 \%$ of $\mathrm{D}_{0} / \mathrm{D}_{5}$-GRP derivative pairs had instrumental response ratio in the range of $0.77-1.3$, and $83 \%$ ones had accurate mass differences with errors $<0.1 \mathrm{mDa}$ compared with its theoretical mass difference value $(\Delta \mathrm{m} / \mathrm{z}=5.031383)$. These results suggested the derivatization method could ensure identification of carbonyl compounds with high reproducibility and accuracy. 
Table S1. Detailed information on compositions of DOM-control samples (disinfectants-before) and experimental samples (disinfectants-after), disinfection types, and reaction duration time.

\begin{tabular}{|c|c|c|c|}
\hline Sample & Composition & Disinfection & Time \\
\hline $\mathrm{O}_{3}$-before & $10.0 \mathrm{mg} \mathrm{C} / \mathrm{L} \mathrm{DOM}, 50 \mathrm{mg} / \mathrm{L} \mathrm{Cl}^{-}$ & NO & 2 hours \\
\hline $\mathrm{O}_{3}$-after & $10.0 \mathrm{mg} \mathrm{C} / \mathrm{L} \mathrm{DOM}, 50 \mathrm{mg} / \mathrm{L} \mathrm{Cl}^{-}$ & ozonation & 2 hours \\
\hline $\mathrm{O}_{3}+\mathrm{Br}$-before & $10.0 \mathrm{mg} \mathrm{C} / \mathrm{L} \mathrm{DOM}, 2 \mathrm{mg} / \mathrm{L} \mathrm{Br}^{-}$ & NO & 2 hours \\
\hline $\mathrm{O}_{3}+\mathrm{Br}$-after & $10.0 \mathrm{mg} \mathrm{C} / \mathrm{L} \mathrm{DOM}, 2 \mathrm{mg} / \mathrm{L} \mathrm{Br}^{-}$ & ozonation & 2 hours \\
\hline $\mathrm{O}_{3}+\mathrm{I}$-before & $10.0 \mathrm{mg} \mathrm{C} / \mathrm{L} \mathrm{DOM}, 2 \mathrm{mg} / \mathrm{L} \mathrm{I}^{-}$ & NO & 2 hours \\
\hline $\mathrm{O}_{3}+\mathrm{I}$-after & $10.0 \mathrm{mg} \mathrm{C} / \mathrm{L}$ DOM, $2 \mathrm{mg} / \mathrm{L} \mathrm{I}^{-}$ & ozonation & 2 hours \\
\hline $\mathrm{NaClO}$-before & $10.0 \mathrm{mg} \mathrm{C} / \mathrm{L}$ DOM & NO & 5 days \\
\hline $\mathrm{NaClO}$-after & $10.0 \mathrm{mg} \mathrm{C} / \mathrm{L}$ DOM & chlorination & 5 days \\
\hline $\mathrm{NaClO}+\mathrm{Br}$-before & $10.0 \mathrm{mg} \mathrm{C} / \mathrm{L} \mathrm{DOM}, 2 \mathrm{mg} / \mathrm{L} \mathrm{Br}^{-}$ & NO & 5 days \\
\hline $\mathrm{NaClO}+\mathrm{Br}$-after & $10.0 \mathrm{mg} \mathrm{C} / \mathrm{L} \mathrm{DOM}, 2 \mathrm{mg} / \mathrm{L} \mathrm{Br}^{-}$ & chlorination & 5 days \\
\hline $\mathrm{NaClO}+\mathrm{I}$-before & $10.0 \mathrm{mg} \mathrm{C} / \mathrm{L} \mathrm{DOM}, 2 \mathrm{mg} / \mathrm{L} \mathrm{I}^{-}$ & NO & 5 days \\
\hline $\mathrm{NaClO}+\mathrm{I}$-after & $10.0 \mathrm{mg} \mathrm{C} / \mathrm{L} \mathrm{DOM}, 2 \mathrm{mg} / \mathrm{L} \mathrm{I}^{-}$ & chlorination & 5 days \\
\hline $\mathrm{NH}_{2} \mathrm{Cl}$-before & $10.0 \mathrm{mg} \mathrm{C} / \mathrm{L} \mathrm{DOM}$ & NO & 1 day \\
\hline $\mathrm{NH}_{2} \mathrm{Cl}$-after & $10.0 \mathrm{mg} \mathrm{C} / \mathrm{L}$ DOM & chloramination & 1 day \\
\hline $\mathrm{NH}_{2} \mathrm{Cl}+\mathrm{Br}$-before & $10.0 \mathrm{mg} \mathrm{C} / \mathrm{L} \mathrm{DOM}, 2 \mathrm{mg} / \mathrm{L} \mathrm{Br}^{-}$ & NO & 1 day \\
\hline $\mathrm{NH}_{2} \mathrm{Cl}+\mathrm{Br}$-after & $10.0 \mathrm{mg} \mathrm{C} / \mathrm{L} \mathrm{DOM}, 2 \mathrm{mg} / \mathrm{L} \mathrm{Br}^{-}$ & chloramination & 1 day \\
\hline $\mathrm{NH}_{2} \mathrm{Cl}+\mathrm{I}$-before & $10.0 \mathrm{mg} \mathrm{C} / \mathrm{L}$ DOM, $2 \mathrm{mg} / \mathrm{L} \mathrm{I}^{-}$ & NO & 1 day \\
\hline $\mathrm{NH}_{2} \mathrm{Cl}+\mathrm{I}$-after & $10.0 \mathrm{mg} \mathrm{C} / \mathrm{L}$ DOM, $2 \mathrm{mg} / \mathrm{L} \mathrm{I}^{-}$ & chloramination & 1 day \\
\hline
\end{tabular}


Table S2. Mass list of known DOM homologous series for internal calibration of FTICR mass spectra in the ESI mode.

\begin{tabular}{llllll}
\hline Formula & Mass $[\mathrm{M}-\mathrm{H}]^{-}$ & Formula & Mass[M-H $]^{-}$ & Formula & Mass[M-H $]^{-}$ \\
\hline $\mathrm{C} 8 \mathrm{H} 10 \mathrm{O} 4$ & 169.05063 & $\mathrm{C} 16 \mathrm{H} 22 \mathrm{O} 6$ & 309.13436 & $\mathrm{C} 22 \mathrm{H} 26 \mathrm{O} 10$ & 449.14532 \\
$\mathrm{C} 9 \mathrm{H} 12 \mathrm{O} 4$ & 183.06628 & $\mathrm{C} 17 \mathrm{H} 24 \mathrm{O} 6$ & 323.15001 & $\mathrm{C} 23 \mathrm{H} 28 \mathrm{O} 10$ & 463.16097 \\
$\mathrm{C} 10 \mathrm{H} 14 \mathrm{O} 4$ & 197.08193 & $\mathrm{C} 16 \mathrm{H} 18 \mathrm{O} 8$ & 337.09289 & $\mathrm{C} 24 \mathrm{H} 30 \mathrm{O} 10$ & 477.17662 \\
$\mathrm{C} 11 \mathrm{H} 16 \mathrm{O} 4$ & 211.09758 & $\mathrm{C} 17 \mathrm{H} 20 \mathrm{O} 8$ & 351.10854 & $\mathrm{C} 25 \mathrm{H} 32 \mathrm{O} 10$ & 491.19227 \\
$\mathrm{C} 12 \mathrm{H} 18 \mathrm{O} 4$ & 225.11323 & $\mathrm{C} 18 \mathrm{H} 22 \mathrm{O} 8$ & 365.12419 & $\mathrm{C} 24 \mathrm{H} 26 \mathrm{O} 12$ & 505.13515 \\
$\mathrm{C} 13 \mathrm{H} 20 \mathrm{O} 4$ & 239.12888 & $\mathrm{C} 19 \mathrm{H} 24 \mathrm{O} 8$ & 379.13984 & $\mathrm{C} 25 \mathrm{H} 28 \mathrm{O} 12$ & 519.15080 \\
$\mathrm{C} 12 \mathrm{H} 14 \mathrm{O} 6$ & 253.07176 & $\mathrm{C} 20 \mathrm{H} 26 \mathrm{O} 8$ & 393.15549 & $\mathrm{C} 26 \mathrm{H} 30 \mathrm{O} 12$ & 533.16645 \\
$\mathrm{C} 13 \mathrm{H} 16 \mathrm{O} 6$ & 267.08741 & $\mathrm{C} 21 \mathrm{H} 28 \mathrm{O} 8$ & 407.17114 & $\mathrm{C} 27 \mathrm{H} 32 \mathrm{O} 12$ & 547.18210 \\
$\mathrm{C} 14 \mathrm{H} 18 \mathrm{O} 6$ & 281.10306 & $\mathrm{C} 20 \mathrm{H} 22 \mathrm{O} 10$ & 421.11402 & $\mathrm{C} 28 \mathrm{H} 34 \mathrm{O} 12$ & 561.19775 \\
$\mathrm{C} 15 \mathrm{H} 20 \mathrm{O} 6$ & 295.11871 & $\mathrm{C} 21 \mathrm{H} 24 \mathrm{O} 10$ & 435.12967 & $\mathrm{C} 29 \mathrm{H} 36 \mathrm{O} 12$ & 575.21340 \\
\hline
\end{tabular}


Table S3. Mass list of known DOM homologous series for internal calibration of FTICR mass spectra in the $\mathrm{ESI}^{+}$mode.

\begin{tabular}{|c|c|c|c|c|c|}
\hline Formula $[\mathrm{M}+\mathrm{H}]^{+}$ & Mass & Formula $[\mathrm{M}+\mathrm{H}]^{+}$ & Mass & Formula $[\mathrm{M}+\mathrm{H}]^{+}$ & Mass \\
\hline $\mathrm{C} 10 \mathrm{H} 15 \mathrm{O} 3$ & 183.10157 & $\mathrm{C} 16 \mathrm{H} 17 \mathrm{O} 8$ & 337.09179 & $\mathrm{C} 24 \mathrm{H} 29 \mathrm{O} 10$ & 477.17552 \\
\hline $\mathrm{C} 11 \mathrm{H} 15 \mathrm{O} 3$ & 195.10157 & C17H19O8 & 351.10744 & C25H31O10 & 491.19117 \\
\hline $\mathrm{C} 10 \mathrm{H} 13 \mathrm{O} 4$ & 197.08084 & $\mathrm{C} 18 \mathrm{H} 21 \mathrm{O} 8$ & 365.12309 & C26H33O10 & 505.20682 \\
\hline $\mathrm{C} 11 \mathrm{H} 17 \mathrm{O} 3$ & 197.11722 & $\mathrm{C} 19 \mathrm{H} 23 \mathrm{O} 8$ & 379.13874 & $\mathrm{C} 27 \mathrm{H} 35 \mathrm{O} 10$ & 519.22247 \\
\hline $\mathrm{C} 10 \mathrm{H} 15 \mathrm{O} 4$ & 199.09649 & $\mathrm{C} 20 \mathrm{H} 25 \mathrm{O} 8$ & 393.15439 & $\mathrm{C} 28 \mathrm{H} 37 \mathrm{O} 10$ & 533.23812 \\
\hline C13H17O5 & 253.10705 & $\mathrm{C} 21 \mathrm{H} 27 \mathrm{O} 8$ & 407.17004 & C19H19O11 & 423.09219 \\
\hline C14H19O5 & 267.12270 & $\mathrm{C} 22 \mathrm{H} 29 \mathrm{O} 8$ & 421.18569 & $\mathrm{C} 20 \mathrm{H} 21 \mathrm{O} 11$ & 437.10784 \\
\hline $\mathrm{C} 15 \mathrm{H} 21 \mathrm{O} 5$ & 281.13835 & $\mathrm{C} 23 \mathrm{H} 31 \mathrm{O} 8$ & 435.20134 & $\mathrm{C} 21 \mathrm{H} 23 \mathrm{O} 11$ & 451.12349 \\
\hline $\mathrm{C} 16 \mathrm{H} 23 \mathrm{O} 5$ & 295.15400 & $\mathrm{C} 24 \mathrm{H} 33 \mathrm{O} 8$ & 449.21699 & $\mathrm{C} 22 \mathrm{H} 25 \mathrm{O} 11$ & 465.13914 \\
\hline $\mathrm{C} 17 \mathrm{H} 25 \mathrm{O} 5$ & 309.16965 & $\mathrm{C} 25 \mathrm{H} 35 \mathrm{O} 8$ & 463.23265 & $\mathrm{C} 23 \mathrm{H} 27 \mathrm{O} 11$ & 479.15479 \\
\hline $\mathrm{C} 18 \mathrm{H} 27 \mathrm{O} 5$ & 323.18530 & $\mathrm{C} 26 \mathrm{H} 37 \mathrm{O} 8$ & 477.24830 & $\mathrm{C} 24 \mathrm{H} 29 \mathrm{O} 11$ & 493.17044 \\
\hline C14H17O6 & 281.10197 & C17H17O9 & 365.08671 & $\mathrm{C} 25 \mathrm{H} 31 \mathrm{O} 11$ & 507.18609 \\
\hline C15H19O6 & 295.11762 & C18H19O9 & 379.10236 & $\mathrm{C} 26 \mathrm{H} 33 \mathrm{O} 11$ & 521.20174 \\
\hline $\mathrm{C} 16 \mathrm{H} 21 \mathrm{O} 6$ & 309.13327 & $\mathrm{C} 19 \mathrm{H} 21 \mathrm{O} 9$ & 393.11801 & $\mathrm{C} 27 \mathrm{H} 35 \mathrm{O} 11$ & 535.21739 \\
\hline $\mathrm{C} 17 \mathrm{H} 23 \mathrm{O} 6$ & 323.14892 & $\mathrm{C} 20 \mathrm{H} 23 \mathrm{O} 9$ & 407.13366 & $\mathrm{C} 28 \mathrm{H} 37 \mathrm{O} 11$ & 549.23304 \\
\hline C18H25O6 & 337.16457 & $\mathrm{C} 21 \mathrm{H} 25 \mathrm{O} 9$ & 421.14931 & $\mathrm{C} 29 \mathrm{H} 39 \mathrm{O} 11$ & 563.24869 \\
\hline $\mathrm{C} 19 \mathrm{H} 27 \mathrm{O} 6$ & 351.18022 & $\mathrm{C} 22 \mathrm{H} 27 \mathrm{O} 9$ & 435.16496 & $\mathrm{C} 21 \mathrm{H} 23 \mathrm{O} 12$ & 467.11840 \\
\hline С20H29O6 & 365.19587 & $\mathrm{C} 23 \mathrm{H} 29 \mathrm{O} 9$ & 449.18061 & $\mathrm{C} 22 \mathrm{H} 25 \mathrm{O} 12$ & 481.13405 \\
\hline $\mathrm{C} 15 \mathrm{H} 17 \mathrm{O} 7$ & 309.09688 & C24H31O9 & 463.19626 & $\mathrm{C} 23 \mathrm{H} 27 \mathrm{O} 12$ & 495.14970 \\
\hline C16H19O7 & 323.11253 & $\mathrm{C} 25 \mathrm{H} 33 \mathrm{O} 9$ & 477.21191 & $\mathrm{C} 24 \mathrm{H} 29 \mathrm{O} 12$ & 509.16535 \\
\hline $\mathrm{C} 17 \mathrm{H} 21 \mathrm{O} 7$ & 337.12818 & $\mathrm{C} 26 \mathrm{H} 35 \mathrm{O} 9$ & 491.22756 & C25H31O12 & 523.18100 \\
\hline $\mathrm{C} 18 \mathrm{H} 23 \mathrm{O} 7$ & 351.14383 & $\mathrm{C} 27 \mathrm{H} 37 \mathrm{O} 9$ & 505.24321 & $\mathrm{C} 26 \mathrm{H} 33 \mathrm{O} 12$ & 537.19665 \\
\hline $\mathrm{C} 19 \mathrm{H} 25 \mathrm{O} 7$ & 365.15948 & C28H39O9 & 519.25886 & $\mathrm{C} 27 \mathrm{H} 35 \mathrm{O} 12$ & 551.21230 \\
\hline $\mathrm{C} 20 \mathrm{H} 27 \mathrm{O} 7$ & 379.17513 & $\mathrm{C} 20 \mathrm{H} 21 \mathrm{O} 10$ & 421.11292 & $\mathrm{C} 28 \mathrm{H} 37 \mathrm{O} 12$ & 565.22795 \\
\hline $\mathrm{C} 21 \mathrm{H} 29 \mathrm{O} 7$ & 393.19078 & $\mathrm{C} 21 \mathrm{H} 23 \mathrm{O} 10$ & 435.12857 & $\mathrm{C} 29 \mathrm{H} 39 \mathrm{O} 12$ & 579.24360 \\
\hline $\mathrm{C} 22 \mathrm{H} 31 \mathrm{O} 7$ & 407.20643 & $\mathrm{C} 22 \mathrm{H} 25 \mathrm{O} 10$ & 449.14422 & & \\
\hline $\mathrm{C} 23 \mathrm{H} 33 \mathrm{O} 7$ & 421.22208 & $\mathrm{C} 23 \mathrm{H} 27 \mathrm{O} 10$ & 463.15987 & & \\
\hline
\end{tabular}


Table S4. An in-house reference mass list of carbonyl compounds for internal calibration of FT-ICR mass spectra in the $\mathrm{ESI}^{+}$mode.

\begin{tabular}{|c|c|c|c|c|c|c|c|}
\hline Formula $[\mathrm{M}]^{+}$ & Mass & Formula $[\mathrm{M}]^{+}$ & Mass & Formula $[\mathrm{M}]^{+}$ & Mass & Formula $[\mathrm{M}]^{+}$ & Mass \\
\hline C7H10N3O & 152.08184 & $16 \mathrm{H} 24 \mathrm{~N} 3 \mathrm{O} 2$ & 90.18630 & $\mathrm{C} 22 \mathrm{H} 34 \mathrm{~N} 3 \mathrm{O} 2$ & 372.26455 & $\mathrm{C} 28 \mathrm{H} 50 \mathrm{~N} 3 \mathrm{O}$ & 444.39484 \\
\hline $\mathrm{C} 8 \mathrm{H} 10 \mathrm{~N} 3 \mathrm{O}$ & 164.08184 & $17 \mathrm{H} 28 \mathrm{~N} 3 \mathrm{O}$ & 90.22269 & $\mathrm{C} 23 \mathrm{H} 38 \mathrm{~N} 3 \mathrm{O}$ & 72.30094 & $\mathrm{C} 26 \mathrm{H} 44 \mathrm{~N} 3 \mathrm{O} 3$ & 446.33772 \\
\hline $\mathrm{C} 9 \mathrm{H} 12 \mathrm{~N} 3 \mathrm{O}$ & 178.09749 & $\mathrm{C} 15 \mathrm{H} 22 \mathrm{~N} 3 \mathrm{O} 3$ & 2.16557 & $\mathrm{C} 21 \mathrm{H} 32 \mathrm{~N} 3 \mathrm{O} 3$ & 4.24382 & $\mathrm{C} 27 \mathrm{H} 48 \mathrm{~N} 3 \mathrm{O} 2$ & 446.37410 \\
\hline $\mathrm{C} 10 \mathrm{H} 14 \mathrm{~N} 3 \mathrm{O}$ & 192.11314 & $\mathrm{C} 16 \mathrm{H} 26 \mathrm{~N} 3 \mathrm{O} 2$ & 292.20195 & $\mathrm{C} 22 \mathrm{H} 36 \mathrm{~N} 3 \mathrm{O} 2$ & 374.28020 & $\mathrm{C} 29 \mathrm{H} 46 \mathrm{~N} 3 \mathrm{O}$ & 452.36354 \\
\hline $\mathrm{C} 9 \mathrm{H} 12 \mathrm{~N} 3 \mathrm{O} 2$ & 194.09240 & $\mathrm{C} 15 \mathrm{H} 24 \mathrm{~N} 3 \mathrm{O} 3$ & 294.18122 & $\mathrm{C} 23 \mathrm{H} 40 \mathrm{~N} 3 \mathrm{O}$ & 374.31659 & $\mathrm{C} 29 \mathrm{H} 48 \mathrm{~N} 3 \mathrm{O}$ & 454.37919 \\
\hline $\mathrm{C} 11 \mathrm{H} 16 \mathrm{~N} 3 \mathrm{O}$ & 206.12879 & $\mathrm{C} 18 \mathrm{H} 24 \mathrm{~N} 3 \mathrm{O}$ & 298.19139 & $\mathrm{C} 21 \mathrm{H} 36 \mathrm{~N} 3 \mathrm{O} 3$ & 8.27512 & $\mathrm{C} 27 \mathrm{H} 42 \mathrm{~N} 3 \mathrm{O} 3$ & 456.32207 \\
\hline $\mathrm{C} 10 \mathrm{H} 14 \mathrm{~N} 3 \mathrm{O} 2$ & 208.10805 & $\mathrm{C} 17 \mathrm{H} 22 \mathrm{~N} 3 \mathrm{O} 2$ & 300.17065 & $\mathrm{C} 24 \mathrm{H} 36 \mathrm{~N} 3 \mathrm{O}$ & 382.28529 & $\mathrm{C} 29 \mathrm{H} 50 \mathrm{~N} 3 \mathrm{O}$ & 456.39484 \\
\hline $\mathrm{C} 12 \mathrm{H} 16 \mathrm{~N} 3 \mathrm{O}$ & 218.12879 & $\mathrm{C} 18 \mathrm{H} 26 \mathrm{~N} 3 \mathrm{O}$ & 300.20704 & $\mathrm{C} 23 \mathrm{H} 34 \mathrm{~N} 3 \mathrm{O} 2$ & 384.26455 & $\mathrm{C} 27 \mathrm{H} 44 \mathrm{~N} 3 \mathrm{O} 3$ & 458.33772 \\
\hline $\mathrm{C} 12 \mathrm{H} 18 \mathrm{~N} 3 \mathrm{O}$ & 220.14444 & $\mathrm{C} 16 \mathrm{H} 20 \mathrm{~N} 3 \mathrm{O} 3$ & 302.14992 & $\mathrm{C} 24 \mathrm{H} 38 \mathrm{~N} 3 \mathrm{O}$ & 384.30094 & $\mathrm{C} 29 \mathrm{H} 52 \mathrm{~N} 3 \mathrm{O}$ & 458.41049 \\
\hline $\mathrm{C} 10 \mathrm{H} 12 \mathrm{~N} 3 \mathrm{O} 3$ & 222.08732 & $\mathrm{C} 17 \mathrm{H} 24 \mathrm{~N} 3 \mathrm{O} 2$ & 302.18630 & $\mathrm{C} 22 \mathrm{H} 32 \mathrm{~N} 3 \mathrm{O} 3$ & 386.24382 & $\mathrm{C} 27 \mathrm{H} 46 \mathrm{~N} 3 \mathrm{O} 3$ & 460.35337 \\
\hline $\mathrm{C} 11 \mathrm{H} 16 \mathrm{~N} 3 \mathrm{O} 2$ & 222.12370 & $\mathrm{C} 18 \mathrm{H} 28 \mathrm{~N} 3 \mathrm{O}$ & 302.22269 & $\mathrm{C} 23 \mathrm{H} 36 \mathrm{~N} 3 \mathrm{O} 2$ & 386.28020 & $\mathrm{C} 28 \mathrm{H} 50 \mathrm{~N} 3 \mathrm{O} 2$ & 460.38975 \\
\hline $\mathrm{C} 13 \mathrm{H} 14 \mathrm{~N} 3 \mathrm{O}$ & 228.11314 & $\mathrm{C} 16 \mathrm{H} 22 \mathrm{~N} 3 \mathrm{O} 3$ & 304.16557 & $\mathrm{C} 24 \mathrm{H} 40 \mathrm{~N} 3 \mathrm{O}$ & 386.31659 & $\mathrm{C} 27 \mathrm{H} 48 \mathrm{~N} 3 \mathrm{O} 3$ & 462.36902 \\
\hline $\mathrm{C} 12 \mathrm{H} 12 \mathrm{~N} 3 \mathrm{O} 2$ & 230.09240 & $\mathrm{C} 17 \mathrm{H} 26 \mathrm{~N} 3 \mathrm{O} 2$ & 304.20195 & $\mathrm{C} 22 \mathrm{H} 34 \mathrm{~N} 3 \mathrm{O} 3$ & 388.25947 & $\mathrm{C} 30 \mathrm{H} 48 \mathrm{~N} 3 \mathrm{O}$ & 466.37919 \\
\hline $\mathrm{C} 13 \mathrm{H} 16 \mathrm{~N} 3 \mathrm{O}$ & 230.12879 & $\mathrm{C} 18 \mathrm{I}$ & 304.23834 & $\mathrm{C} 23 \mathrm{H} 38 \mathrm{~N}$ & 85 & $\mathrm{C} 30 \mathrm{H} 5$ & \\
\hline $\mathrm{C} 13 \mathrm{H} 18 \mathrm{~N} 3 \mathrm{O}$ & 232.14444 & $\mathrm{C} 17 \mathrm{H} 28 \mathrm{~N} 3 \mathrm{O} 2$ & 306.21760 & $\mathrm{C} 24 \mathrm{H} 42 \mathrm{~N} 3 \mathrm{O}$ & 388.33224 & $\mathrm{C} 30 \mathrm{H} 52 \mathrm{~N} 3 \mathrm{O}$ & 470.41049 \\
\hline $\mathrm{C} 12 \mathrm{H} 16 \mathrm{~N} 3 \mathrm{O} 2$ & 234.12370 & $\mathrm{C} 16 \mathrm{H} 26 \mathrm{~N} 3 \mathrm{O} 3$ & 308.19687 & $\mathrm{C} 22 \mathrm{H} 36 \mathrm{~N} 3 \mathrm{O} 3$ & 390.27512 & $\mathrm{C} 28 \mathrm{H} 46 \mathrm{~N} 3 \mathrm{O} 3$ & 472.35337 \\
\hline $\mathrm{C} 13 \mathrm{H} 20 \mathrm{~N} 3 \mathrm{O}$ & 234.16009 & $\mathrm{C} 19 \mathrm{H} 26 \mathrm{~N} 3 \mathrm{O}$ & 312.20704 & $\mathrm{C} 23 \mathrm{H} 40 \mathrm{~N} 3 \mathrm{O} 2$ & 50 & $\mathrm{C} 29 \mathrm{H} 50 \mathrm{~N}$ & 472.38975 \\
\hline $\mathrm{C} 11 \mathrm{H} 14 \mathrm{~N} 3 \mathrm{O} 3$ & 236.10297 & $\mathrm{C} 18 \mathrm{H} 24 \mathrm{~N} 3 \mathrm{O} 2$ & 314.18630 & $\mathrm{C} 22 \mathrm{H} 38 \mathrm{~N} 3 \mathrm{O} 3$ & 392.29077 & $\mathrm{C} 30 \mathrm{H} 54 \mathrm{~N} 3 \mathrm{O}$ & 472.42614 \\
\hline $\mathrm{C} 12 \mathrm{H} 18 \mathrm{~N} 3 \mathrm{O} 2$ & 236.13935 & $\mathrm{C} 17 \mathrm{H} 22 \mathrm{~N} 3 \mathrm{O} 3$ & 316.16557 & $\mathrm{C} 25 \mathrm{H} 38 \mathrm{~N} 3 \mathrm{O}$ & 396.30094 & $\mathrm{C} 28 \mathrm{H} 48 \mathrm{~N} 3 \mathrm{O} 3$ & 474.36902 \\
\hline $\mathrm{C} 13 \mathrm{H} 14 \mathrm{~N} 3 \mathrm{O} 2$ & 244.10805 & $\mathrm{C} 18 \mathrm{H} 26 \mathrm{~N} 3 \mathrm{O} 2$ & 316.20195 & $\mathrm{C} 24 \mathrm{H} 36 \mathrm{~N} 3 \mathrm{O} 2$ & 398.28020 & $\mathrm{C} 28 \mathrm{H} 50 \mathrm{~N} 3 \mathrm{O} 3$ & \\
\hline $\mathrm{C} 14 \mathrm{H} 18 \mathrm{~N} 3 \mathrm{O}$ & 244.14444 & C19H30N3O & 316.23834 & $\mathrm{C} 25 \mathrm{H} 40 \mathrm{~N} 3 \mathrm{O}$ & 659 & $\mathrm{C} 31 \mathrm{H} 52 \mathrm{~N} 3 \mathrm{O}$ & 1049 \\
\hline $\mathrm{C} 13 \mathrm{H} 16 \mathrm{~N} 3 \mathrm{O} 2$ & 246.12370 & $\mathrm{C} 17 \mathrm{H} 24 \mathrm{~N} 3 \mathrm{O} 3$ & 318.18122 & $\mathrm{C} 23 \mathrm{H} 34 \mathrm{~N} 3 \mathrm{O} 3$ & 400.25947 & $\mathrm{C} 31 \mathrm{H} 54 \mathrm{~N} 3 \mathrm{O}$ & 484.42614 \\
\hline $\mathrm{C} 14 \mathrm{H} 20 \mathrm{~N} 3 \mathrm{O}$ & 246.16009 & $\mathrm{C} 18 \mathrm{H} 28 \mathrm{~N} 3 \mathrm{O} 2$ & 318.21760 & $\mathrm{C} 25 \mathrm{H} 42 \mathrm{~N} 3 \mathrm{O}$ & 400.33 & $\mathrm{C} 29 \mathrm{H} 48$ & 6902 \\
\hline $\mathrm{C} 13 \mathrm{H} 18 \mathrm{~N} 3 \mathrm{O} 2$ & 248.13935 & $\mathrm{C} 19 \mathrm{H} 32 \mathrm{~N} 3 \mathrm{O}$ & 318.25399 & $\mathrm{C} 23 \mathrm{H} 36 \mathrm{~N} 3 \mathrm{O} 3$ & 402.27512 & $\mathrm{C} 30 \mathrm{H} 52 \mathrm{~N} 3 \mathrm{O} 2$ & 486.40540 \\
\hline $\mathrm{C} 14 \mathrm{H} 22 \mathrm{~N} 3 \mathrm{O}$ & 248.17574 & $\mathrm{C} 17 \mathrm{H} 26 \mathrm{~N} 3 \mathrm{O} 3$ & 320.19687 & $\mathrm{C} 24 \mathrm{H} 40 \mathrm{~N} 3 \mathrm{O} 2$ & 402.31150 & $\mathrm{C} 31 \mathrm{H} 5$ & 486.44179 \\
\hline $\mathrm{C} 12 \mathrm{H} 16 \mathrm{~N} 3 \mathrm{O} 3$ & 250.11862 & $\mathrm{C} 18 \mathrm{H}$ & 320.23325 & $\mathrm{C} 25 \mathrm{H} 44$ & 89 & $\mathrm{C} 2 \mathrm{~S}$ & 467 \\
\hline $\mathrm{C} 13 \mathrm{H} 20 \mathrm{~N} 3 \mathrm{O} 2$ & 250.15500 & $\mathrm{C} 20 \mathrm{H}$ & 2269 & $\mathrm{C} 23 \mathrm{H} 38 \mathrm{~N} 3 \mathrm{O} 3$ & 777 & $\mathrm{C} 29 \mathrm{H} 52 \mathrm{~N}$ & 032 \\
\hline $\mathrm{C} 15 \mathrm{H} 18 \mathrm{~N} 3 \mathrm{O}$ & 256.14444 & $\mathrm{C} 19 \mathrm{H} 26 \mathrm{~N} 3 \mathrm{O} 2$ & 328.20195 & $\mathrm{C} 23 \mathrm{H} 40$ & 406 & $\mathrm{C} 30 \mathrm{H} 48 \mathrm{I}$ & 498.36902 \\
\hline $\mathrm{C} 14 \mathrm{H} 16 \mathrm{~N} 3 \mathrm{O} 2$ & 258.12370 & $\mathrm{C} 18 \mathrm{H} 24 \mathrm{~N} 3 \mathrm{O} 3$ & 330.18122 & $\mathrm{C} 26 \mathrm{H} 42 \mathrm{~N} 3 \mathrm{O}$ & 412.3 & C31H52N & 498.40540 \\
\hline $\mathrm{C} 15 \mathrm{H} 20 \mathrm{~N} 3 \mathrm{O}$ & 258.16009 & $\mathrm{C} 19 \mathrm{H}$ & & $\mathrm{C} 24 \mathrm{H} 36 \mathrm{~N} 3 \mathrm{O} 3$ & & $\mathrm{C} 32 \mathrm{H} 56 \mathrm{I}$ & 498.44179 \\
\hline $\mathrm{C} 14 \mathrm{H} 18 \mathrm{~N} 3 \mathrm{O} 2$ & 260.13935 & $\mathrm{C} 20 \mathrm{H} 32 \mathrm{~N} 3 \mathrm{O}$ & 330.25399 & $\mathrm{C} 25 \mathrm{H} 40 \mathrm{~N} 3 \mathrm{O} 2$ & 150 & $\mathrm{C} 31 \mathrm{H} 54 \mathrm{~N} 3 \mathrm{O} 2$ & 500.42105 \\
\hline $\mathrm{C} 15 \mathrm{H} 22 \mathrm{~N} 3 \mathrm{O}$ & 260.17574 & $\mathrm{C} 18 \mathrm{H} 26 \mathrm{~N} 3 \mathrm{O} 3$ & 332.19687 & $\mathrm{C} 26 \mathrm{H} 44 \mathrm{~N} 3 \mathrm{O}$ & 414.34 & $\mathrm{C} 32 \mathrm{H} 58 \mathrm{~N} 3 \mathrm{O}$ & 500.45744 \\
\hline $\mathrm{C} 13 \mathrm{H} 16 \mathrm{~N} 3 \mathrm{O} 3$ & 262.11862 & $\mathrm{C} 19 \mathrm{H} 30 \mathrm{~N} 3 \mathrm{O} 2$ & & $\mathrm{C} 24 \mathrm{H} 38 \mathrm{~N} 3 \mathrm{O} 3$ & & $\mathrm{C} 31 \mathrm{H} 56 \mathrm{~N} 3 \mathrm{O} 2$ & 3670 \\
\hline $\mathrm{C} 14 \mathrm{H} 20 \mathrm{~N} 3 \mathrm{O} 2$ & 262.15500 & $\mathrm{C} 20 \mathrm{H} 34 \mathrm{~N} 3 \mathrm{O}$ & 332.26964 & $\mathrm{C} 25 \mathrm{H} 42 \mathrm{~N} 3 \mathrm{O} 2$ & 715 & C33H56N3O & 510.44179 \\
\hline $\mathrm{C} 15 \mathrm{H} 24 \mathrm{~N} 3 \mathrm{O}$ & 262.19139 & $\mathrm{C} 18 \mathrm{H} 28 \mathrm{~N} 3 \mathrm{O} 3$ & 334.21252 & $\mathrm{C} 26 \mathrm{H} 46 \mathrm{~N} 3 \mathrm{O}$ & 416.36354 & $\mathrm{C} 32 \mathrm{H} 54 \mathrm{~N} 3 \mathrm{O} 2$ & 512.42105 \\
\hline $\mathrm{C} 13 \mathrm{H} 18 \mathrm{~N} 3 \mathrm{O} 3$ & & $\mathrm{C} 19 \mathrm{H} 3$ & & $\mathrm{C} 24 \mathrm{H} 40 \mathrm{~N} 3 \mathrm{O} 3$ & & $\mathrm{C} 33 \mathrm{H} 58 \mathrm{~N} 3 \mathrm{O}$ & 512.45744 \\
\hline $\mathrm{C} 14 \mathrm{H} 22 \mathrm{~N} 3 \mathrm{O} 2$ & 264.17065 & $\mathrm{C} 21 \mathrm{H} 30 \mathrm{~N} 3 \mathrm{O}$ & 340.23834 & $\mathrm{C} 25 \mathrm{H} 44 \mathrm{~N} 3 \mathrm{O} 2$ & 418.34280 & $\mathrm{C} 31 \mathrm{H} 52 \mathrm{~N} 3 \mathrm{O} 3$ & 514.40032 \\
\hline $\mathrm{C} 13 \mathrm{H} 20 \mathrm{~N} 3 \mathrm{O} 3$ & 266.14992 & $\mathrm{C} 21 \mathrm{H} 32 \mathrm{~N} 3 \mathrm{O}$ & 342.25399 & $\mathrm{C} 24 \mathrm{H} 42 \mathrm{~N} 3 \mathrm{O} 3$ & 420.32207 & C33H60N3O & 514.47309 \\
\hline $\mathrm{C} 16 \mathrm{H} 20 \mathrm{~N} 3 \mathrm{O}$ & 270.16009 & C19H26N3O3 & 344.19687 & $\mathrm{C} 27 \mathrm{H} 42 \mathrm{~N} 3 \mathrm{O}$ & 424.33224 & C31H54N3O3 & 516.41597 \\
\hline $\mathrm{C} 15 \mathrm{H} 18 \mathrm{~N} 3 \mathrm{O} 2$ & 272.13935 & $\mathrm{C} 20 \mathrm{H} 30 \mathrm{~N} 3 \mathrm{O} 2$ & 344.23325 & $\mathrm{C} 26 \mathrm{H} 40 \mathrm{~N} 3 \mathrm{O} 2$ & 426.31150 & $\mathrm{C} 31 \mathrm{H} 56 \mathrm{~N} 3 \mathrm{O} 3$ & 518.43162 \\
\hline $\mathrm{C} 16 \mathrm{H} 22 \mathrm{~N} 3 \mathrm{O}$ & 272.17574 & $\mathrm{C} 21 \mathrm{H} 34 \mathrm{~N} 3 \mathrm{O}$ & 344.26964 & $\mathrm{C} 27 \mathrm{H} 44 \mathrm{~N} 3 \mathrm{O}$ & 426.34789 & C34H60N3O & 526.47309 \\
\hline $\mathrm{C} 14 \mathrm{H} 16 \mathrm{~N} 3 \mathrm{O} 3$ & 274.11862 & $\mathrm{C} 19 \mathrm{H} 28 \mathrm{~N} 3 \mathrm{O} 3$ & 346.21252 & $\mathrm{C} 25 \mathrm{H} 38 \mathrm{~N} 3 \mathrm{O} 3$ & 428.29077 & $\mathrm{C} 33 \mathrm{H} 58 \mathrm{~N} 3 \mathrm{O} 2$ & 528.45236 \\
\hline $\mathrm{C} 15 \mathrm{H} 20 \mathrm{~N} 3 \mathrm{O} 2$ & 274.15500 & $\mathrm{C} 20 \mathrm{H} 32 \mathrm{~N} 3 \mathrm{O} 2$ & 346.24890 & $\mathrm{C} 26 \mathrm{H} 42 \mathrm{~N} 3 \mathrm{O} 2$ & 428.32715 & $\mathrm{C} 34 \mathrm{H} 62 \mathrm{~N} 3 \mathrm{O}$ & 528.48874 \\
\hline $\mathrm{C} 16 \mathrm{H} 24 \mathrm{~N} 3 \mathrm{O}$ & 274.19139 & $\mathrm{C} 21 \mathrm{H} 36 \mathrm{~N} 3 \mathrm{O}$ & 346.28529 & $\mathrm{C} 27 \mathrm{H} 46 \mathrm{~N} 3 \mathrm{O}$ & 428.36354 & $\mathrm{C} 32 \mathrm{H} 56 \mathrm{~N} 3 \mathrm{O} 3$ & 530.43162 \\
\hline $\mathrm{C} 14 \mathrm{H} 18 \mathrm{~N} 3 \mathrm{O} 3$ & 276.13427 & $\mathrm{C} 20 \mathrm{H} 34 \mathrm{~N} 3 \mathrm{O} 2$ & 348.26455 & $\mathrm{C} 25 \mathrm{H} 40 \mathrm{~N} 3 \mathrm{O} 3$ & 430.30642 & $\mathrm{C} 35 \mathrm{H} 62 \mathrm{~N} 3 \mathrm{O}$ & 540.48874 \\
\hline $\mathrm{C} 15 \mathrm{H} 22 \mathrm{~N} 3 \mathrm{O} 2$ & 276.17065 & $\mathrm{C} 22 \mathrm{H} 32 \mathrm{~N} 3 \mathrm{O}$ & 354.25399 & $\mathrm{C} 26 \mathrm{H} 44 \mathrm{~N} 3 \mathrm{O} 2$ & 430.34280 & $\mathrm{C} 33 \mathrm{H} 56 \mathrm{~N} 3 \mathrm{O} 3$ & 542.43162 \\
\hline $\mathrm{C} 16 \mathrm{H} 26 \mathrm{~N} 3 \mathrm{O}$ & 276.20704 & $\mathrm{C} 22 \mathrm{H} 34 \mathrm{~N} 3 \mathrm{O}$ & 356.26964 & $\mathrm{C} 27 \mathrm{H} 48 \mathrm{~N} 3 \mathrm{O}$ & 430.37919 & $\mathrm{C} 35 \mathrm{H} 64 \mathrm{~N} 3 \mathrm{O}$ & 542.50439 \\
\hline $3 \mathrm{O} 3$ & 278.14992 & $\mathrm{C} 21 \mathrm{H} 32 \mathrm{~N} 3 \mathrm{O} 2$ & 358.24890 & $\mathrm{C} 25 \mathrm{H} 42 \mathrm{~N} 3 \mathrm{O} 3$ & 432.32207 & $\mathrm{C} 33 \mathrm{H} 58 \mathrm{~N} 3 \mathrm{O} 3$ & 544.44727 \\
\hline $\mathrm{C} 15 \mathrm{H} 24 \mathrm{~N} 3 \mathrm{O} 2$ & 278.18630 & $\mathrm{C} 22 \mathrm{H} 36 \mathrm{~N} 3 \mathrm{O}$ & 358.28529 & $\mathrm{C} 25 \mathrm{H} 44 \mathrm{~N} 3 \mathrm{O} 3$ & 434.33772 & $\mathrm{C} 36 \mathrm{H} 64 \mathrm{~N} 3 \mathrm{O}$ & 554.50439 \\
\hline $\mathrm{C} 14 \mathrm{H} 22 \mathrm{~N} 3 \mathrm{O} 3$ & 280.16557 & $\mathrm{C} 20 \mathrm{H} 30 \mathrm{~N} 3 \mathrm{O} 3$ & 360.22817 & $\mathrm{C} 28 \mathrm{H} 44 \mathrm{~N} 3 \mathrm{O}$ & 438.34789 & $\mathrm{C} 34 \mathrm{H} 58 \mathrm{~N} 3 \mathrm{O} 3$ & 556.44727 \\
\hline $\mathrm{C} 17 \mathrm{H} 22 \mathrm{~N} 3 \mathrm{O}$ & 284.17574 & $\mathrm{C} 21 \mathrm{H} 34 \mathrm{~N} 3 \mathrm{O} 2$ & 360.26455 & $\mathrm{C} 27 \mathrm{H} 42 \mathrm{~N} 3 \mathrm{O} 2$ & 440.32715 & $\mathrm{C} 35 \mathrm{H} 62 \mathrm{~N} 3 \mathrm{O} 2$ & 556.48366 \\
\hline $\mathrm{C} 16 \mathrm{H} 20 \mathrm{~N} 3 \mathrm{O} 2$ & 286.15500 & $\mathrm{C} 22 \mathrm{H} 38 \mathrm{~N} 3 \mathrm{O}$ & 360.30094 & $\mathrm{C} 28 \mathrm{H} 46 \mathrm{~N} 3 \mathrm{O}$ & 440.36354 & C36H66N3O & 556.52004 \\
\hline $\mathrm{C} 17 \mathrm{H} 24 \mathrm{~N} 3 \mathrm{O}$ & 286.19139 & $\mathrm{C} 2 \mathrm{OH} 32 \mathrm{~N} 3 \mathrm{O} 3$ & 362.24382 & $\mathrm{C} 26 \mathrm{H} 40 \mathrm{~N} 3 \mathrm{O} 3$ & 442.30642 & $\mathrm{C} 37 \mathrm{H} 66 \mathrm{~N} 3 \mathrm{O}$ & 568.52004 \\
\hline $\mathrm{C} 15 \mathrm{H} 18 \mathrm{~N} 3 \mathrm{O} 3$ & 288.13427 & $\mathrm{C} 21 \mathrm{H} 36 \mathrm{~N} 3 \mathrm{O} 2$ & 362.28020 & $\mathrm{C} 27 \mathrm{H} 44 \mathrm{~N} 3 \mathrm{O} 2$ & 442.34280 & C37H68N3O & 570.53569 \\
\hline $\mathrm{C} 16 \mathrm{H} 22 \mathrm{~N} 3 \mathrm{O} 2$ & 288.17065 & $\mathrm{C} 23 \mathrm{H} 34 \mathrm{~N} 3 \mathrm{O}$ & 368.26964 & $\mathrm{C} 28 \mathrm{H} 48 \mathrm{~N} 3 \mathrm{O}$ & 442.37919 & $\mathrm{C} 38 \mathrm{H} 70 \mathrm{~N} 3 \mathrm{O}$ & 584.55134 \\
\hline $\mathrm{C} 17 \mathrm{H} 26 \mathrm{~N} 3 \mathrm{O}$ & 288.20704 & $\mathrm{C} 22 \mathrm{H} 32 \mathrm{~N} 3 \mathrm{O} 2$ & 370.24890 & $\mathrm{C} 26 \mathrm{H} 42 \mathrm{~N} 3 \mathrm{O} 3$ & 444.32207 & $\mathrm{C} 39 \mathrm{H} 72 \mathrm{~N} 3 \mathrm{O}$ & 598.56699 \\
\hline $\mathrm{C} 15 \mathrm{H} 20 \mathrm{~N} 3 \mathrm{O} 3$ & 290.14992 & $\mathrm{C} 23 \mathrm{H} 36 \mathrm{~N} 3 \mathrm{O}$ & 370.28529 & $\mathrm{C} 27 \mathrm{H} 46 \mathrm{~N} 3 \mathrm{O} 2$ & 444.35845 & $\mathrm{C} 40 \mathrm{H} 72 \mathrm{~N} 3 \mathrm{O}$ & 610.56699 \\
\hline
\end{tabular}


Table S5. Magnitude-weighted average values of molecular characteristics of carbonyl compounds generated at the varied derivatization reaction temperatures.

\begin{tabular}{cccccc}
\hline Temperature $\left({ }^{\circ} \mathrm{C}\right)$ & $\mathrm{O} / \mathrm{C}_{\mathrm{w}}$ & $\mathrm{H} / \mathrm{C}_{\mathrm{w}}$ & $\mathrm{DBE}_{\mathrm{w}}$ & $\mathrm{DBE} / \mathrm{C}_{\mathrm{w}}$ & $\mathrm{MW}_{\mathrm{w}}$ \\
\hline 30 & $0.47 \pm 0.00$ & $1.23 \pm 0.00$ & $6.83 \pm 0.10$ & $0.46 \pm 0.00$ & $311.0 \pm 4.7$ \\
40 & $0.47 \pm 0.00$ & $1.22 \pm 0.01$ & $6.74 \pm 0.03$ & $0.46 \pm 0.00$ & $304.9 \pm 1.9$ \\
50 & $0.47 \pm 0.00$ & $1.23 \pm 0.00$ & $6.83 \pm 0.08$ & $0.46 \pm 0.00$ & $307.8 \pm 3.4$ \\
60 & $0.46 \pm 0.00$ & $1.22 \pm 0.00$ & $7.08 \pm 0.01$ & $0.46 \pm 0.00$ & $319.8 \pm 0.5$ \\
\hline
\end{tabular}


Table S6. Magnitude-weighted average values of molecular characteristics of CHO carbonyl compounds before and after disinfection (ozonation, chlorination and chloramination).

\begin{tabular}{lcccccccc}
\hline Sample & $\mathrm{C}_{\mathrm{w}}$ & $\mathrm{H}_{\mathrm{w}}$ & $\mathrm{O}_{\mathrm{w}}$ & $\mathrm{O} \mathrm{C}_{\mathrm{w}}$ & $\mathrm{H} \mathrm{C}_{\mathrm{w}}$ & $\mathrm{DBE}_{\mathrm{w}}$ & ${\mathrm{DBE} / \mathrm{C}_{\mathrm{w}}}$ & $\mathrm{MW}_{\mathrm{w}}$ \\
\hline $\mathrm{O}_{3}$-Before & 18.3 & 21.9 & 8.1 & 0.45 & 1.19 & 8.33 & 0.46 & 371.0 \\
$\mathrm{O}_{3}$-After & 15.1 & 18.2 & 8.1 & 0.54 & 1.20 & 7.03 & 0.47 & 328.8 \\
NaClO-Before & 18.2 & 21.8 & 7.9 & 0.44 & 1.19 & 8.27 & 0.46 & 366.0 \\
$\mathrm{NaClO}-$ After & 17.4 & 21.3 & 7.5 & 0.44 & 1.22 & 7.71 & 0.45 & 349.5 \\
$\mathrm{NH}_{2}$ Cl-Before & 18.3 & 21.9 & 8.0 & 0.45 & 1.19 & 8.33 & 0.46 & 369.6 \\
$\mathrm{NH}_{2} \mathrm{Cl}$-After & 17.1 & 20.5 & 7.9 & 0.47 & 1.19 & 7.85 & 0.47 & 351.4 \\
\hline
\end{tabular}


Table S7. A summary of the halogenated carbonyl DBPs reported in literature (with only one possible isomer shown in the table).

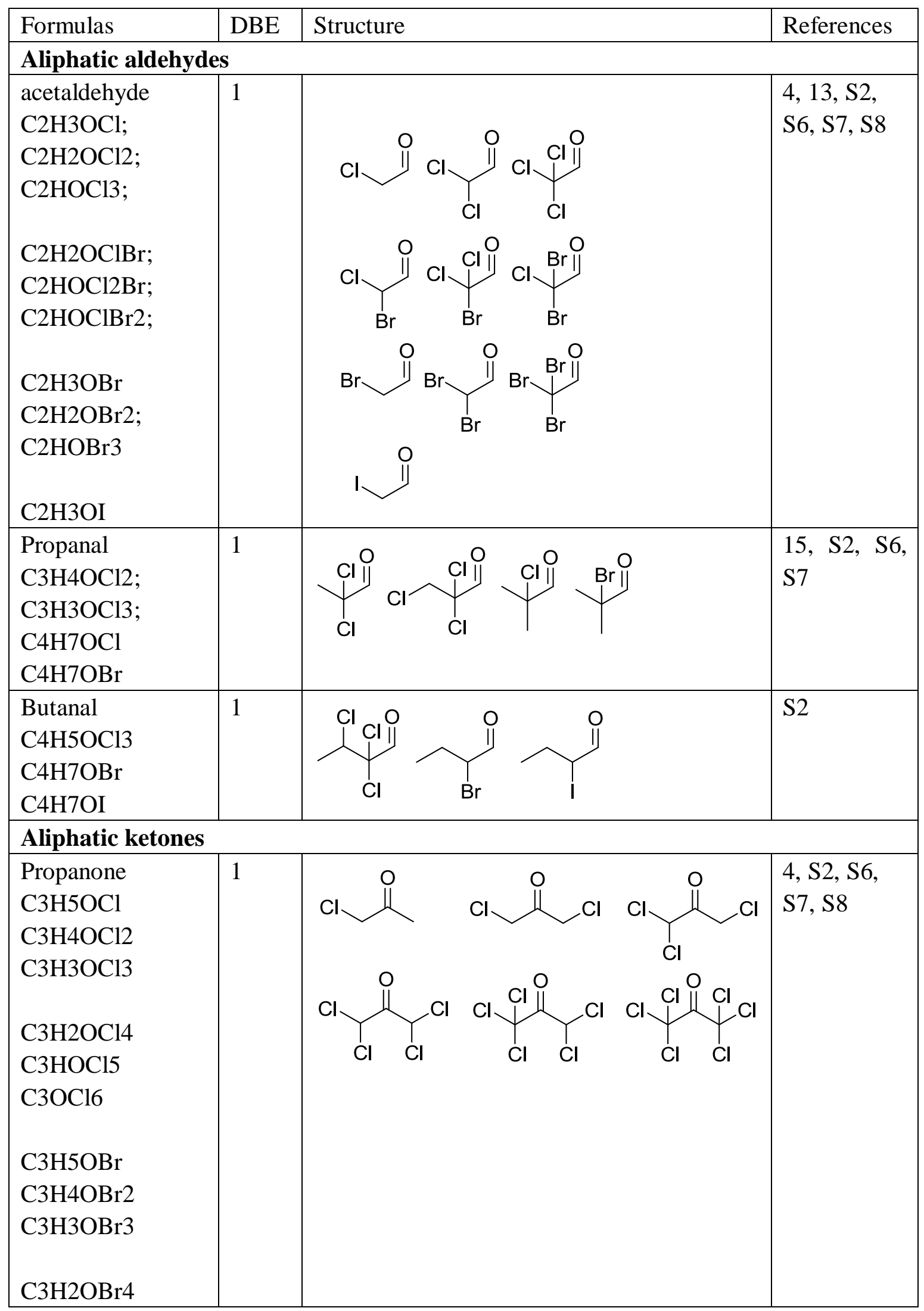




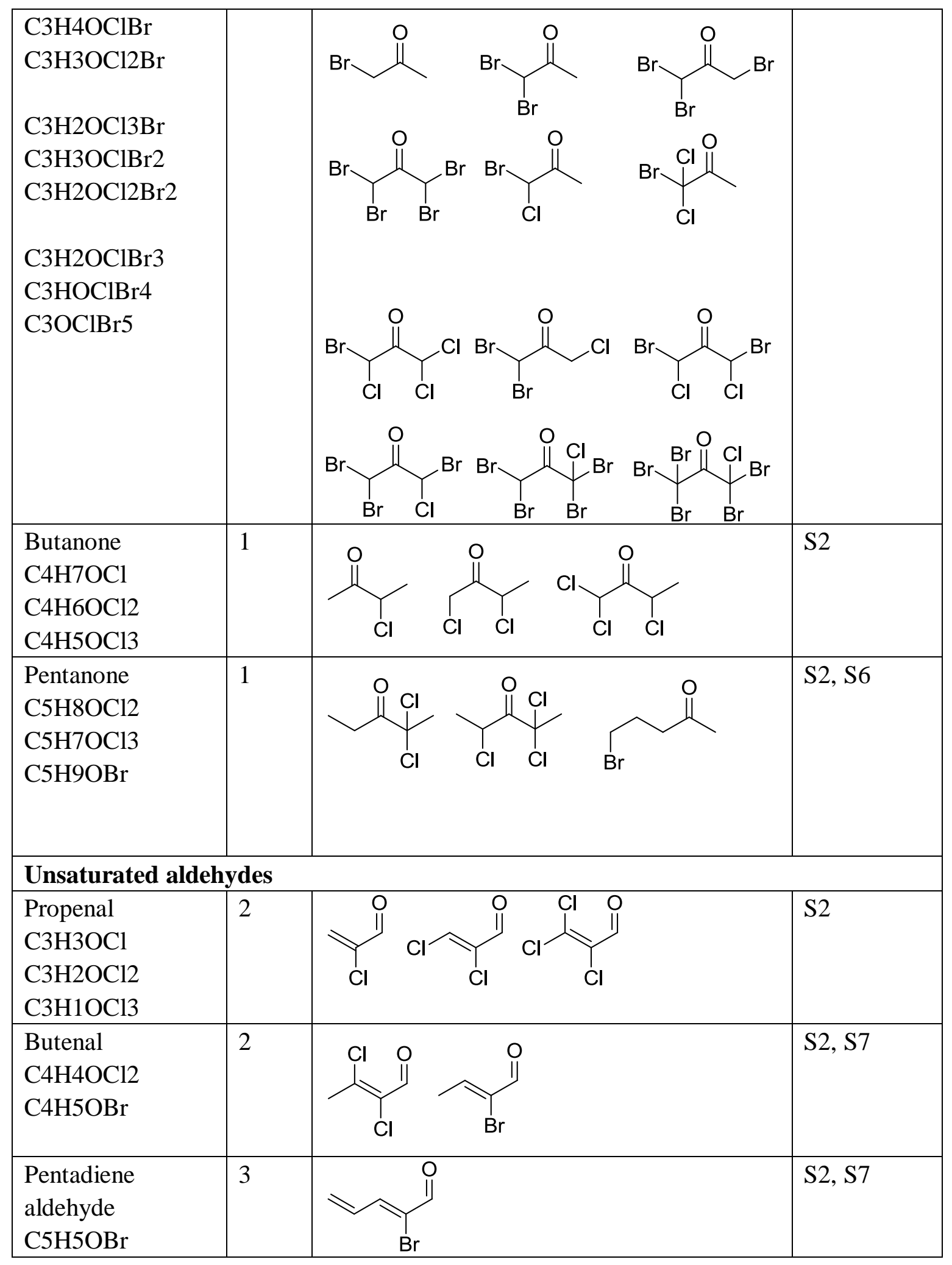




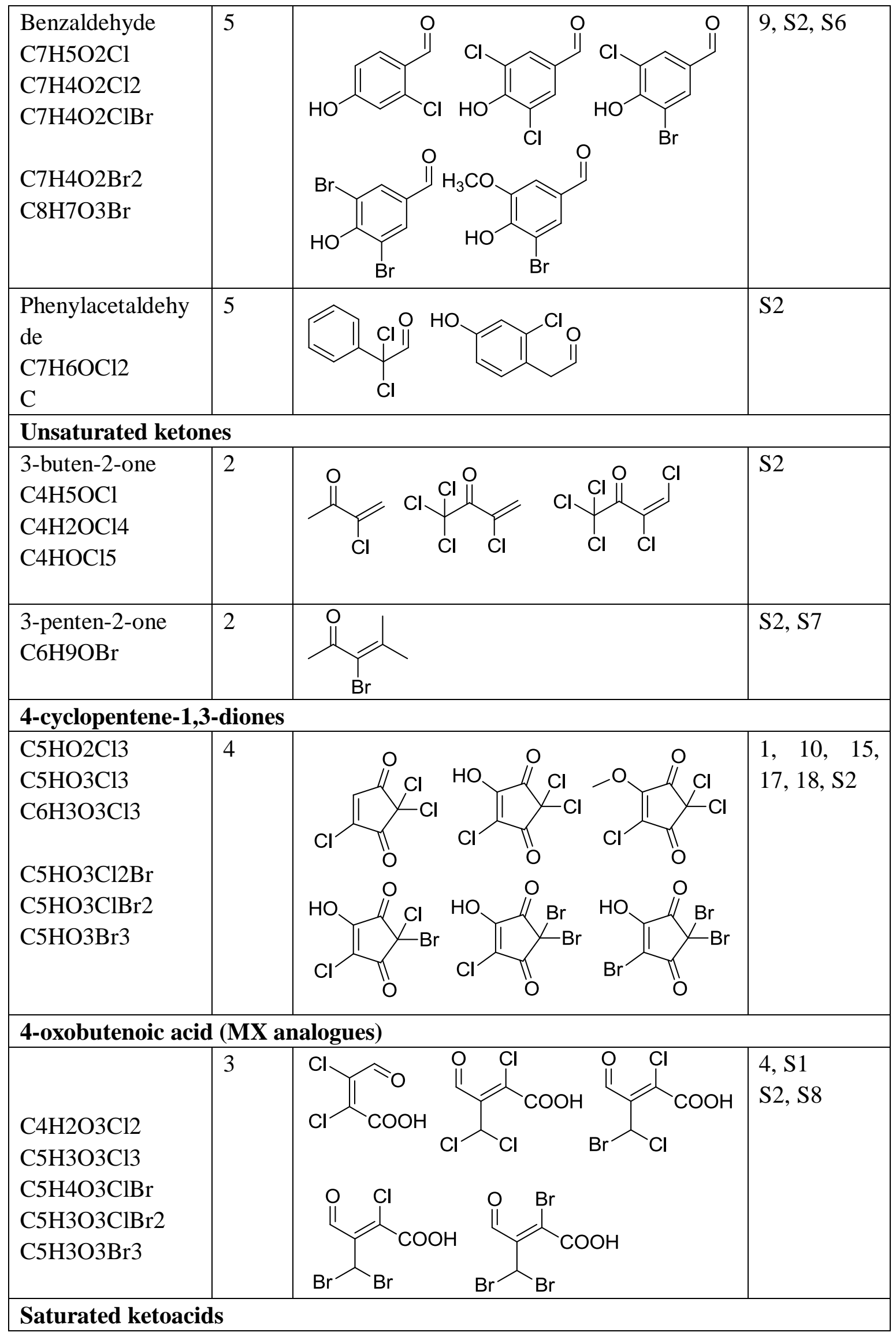




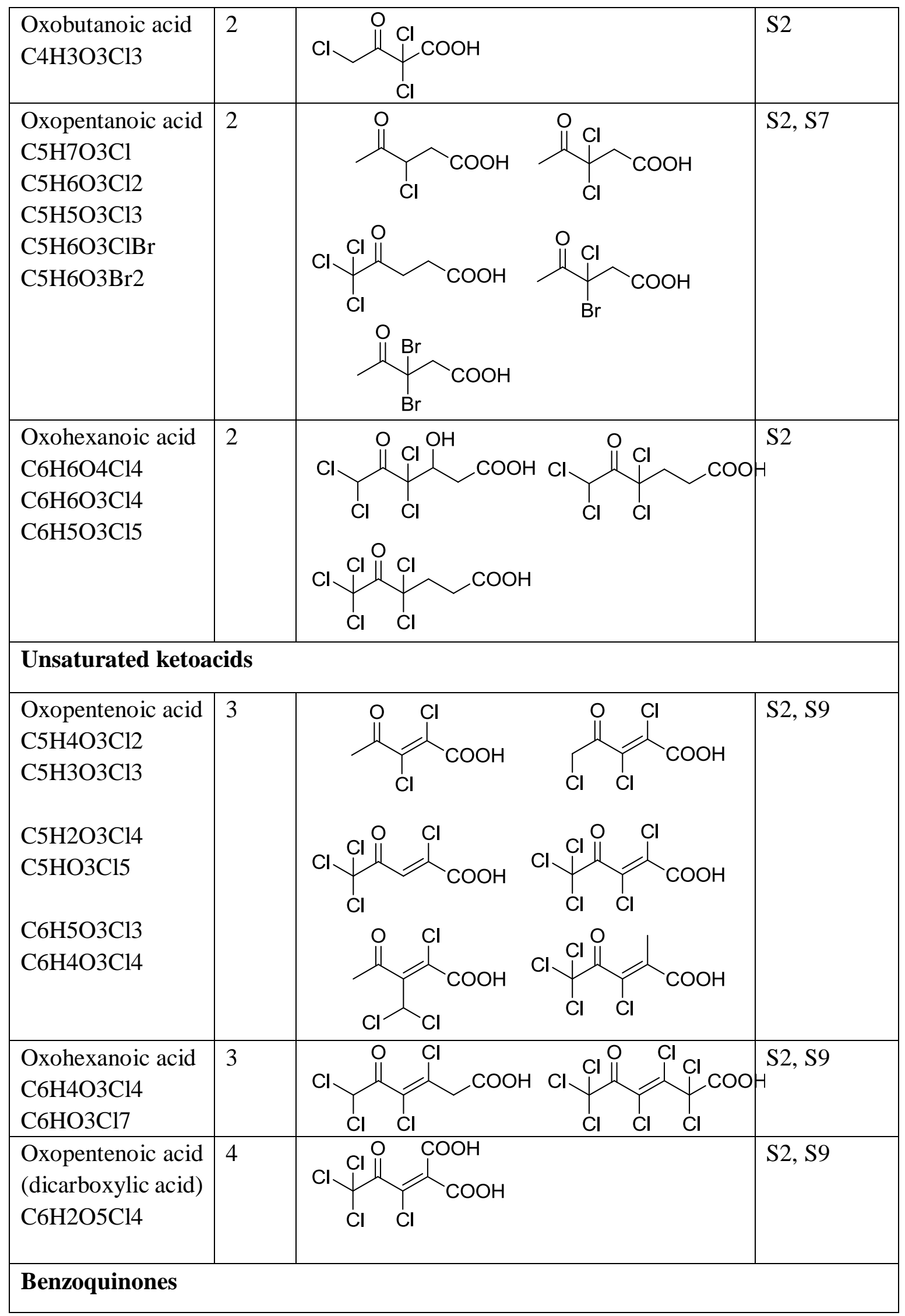




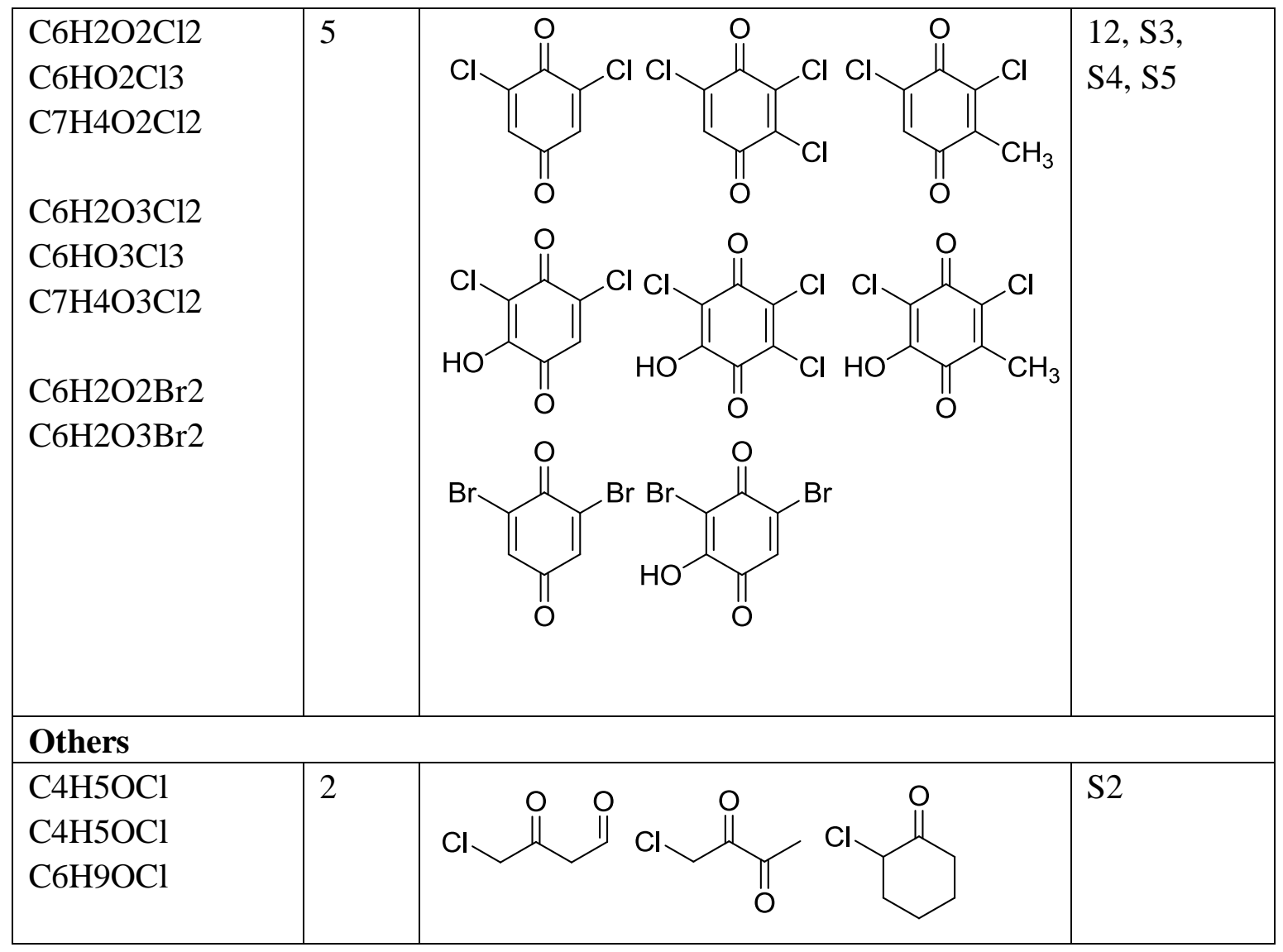

\section{Relevant references in the main text}

[1]. Gonsior, M.; Schmitt-Kopplin, P.; Stavklint, H.; Richardson, S. D.; Hertkorn, N.; Bastviken, D. Changes in dissolved organic matter during the treatment processes of a drinking water plant in Sweden and formation of previously unknown disinfection byproducts. Environ. Sci. Technol. 2014, 48 (21), 12714-12722.

[4]. Krasner, S. W.; Weinberg, H. S.; Richardson, S. D.; Pastor, S. J.; Chinn, R.; Sclimenti, M. J.; Onstad, G. D.; Thruston, A. D. Occurrence of a new generation of disinfection byproducts. Environ. Sci. Technol. 2006, 40 (23), 7175-7185.

[9]. Pan, Y.; Zhang, X. R. Four groups of new aromatic halogenated disinfection byproducts: Effect of bromide concentration on their formation and speciation in chlorinated drinking water. Environ. Sci. Technol. 2013, 47 (3), 1265-1273.

[10]. Zhai, H. Y.; Zhang, X. R.; Zhu, X. H.; Liu, J. Q.; Ji, M. Formation of brominated disinfection byproducts during chloramination of drinking water: New polar species and overall kinetics. Environ. Sci. Technol. 2014, 48 (5), 2579-2588.

[12]. Qin, F.; Zhao, Y. Y.; Zhao, Y. L.; Boyd, J. M.; Zhou, W. J.; Li, X. F. A toxic disinfection byproduct, 2,6-dichloro-1,4-benzoquinone, identified in drinking water. Angew. Chem. Int. Ed. 2010, 49 (4), 790-892.

[13]. Jeong, C. H.; Postigo, C.; Richardson, S. D.; Simmons, J. E.; Kimura, S. Y.; Marinas, B. J.; Barcelo, D.; Liang, P.; Wagner, E. D.; Plewa, M. J. Occurrence and comparative toxicity of haloacetaldehyde disinfection byproducts in drinking water. Environ. Sci. Technol. 2015, 49 (23), 13749-13759. 
[15].Richardson, S. D.; Thruston, A. D.; Caughran, T. V.; Chen, P. H.; Collette, T. W.; Floyd, T. L.; Schenck, K. M.; Lykins, B. W.; Sun, G. R.; Majetich, G. Identification of new ozone disinfection byproducts in drinking water. Environ. Sci. Technol 1999, 33 (19), 3368-3377.

[17]. Pan, Y.; Li, W. B.; Li, A. M.; Zhou, Q.; Shi, P.; Wang, Y. A new group of disinfection byproducts in drinking water: Trihalo-hydroxy-cyclopentene-diones. Environ. Sci. Technol. 2016, 50 (14), 7344-7352.

[18]. Gong, H. J.; You, Z.; Xian, Q. M.; Shen, X.; Zou, H. X.; Huan, F.; Xu, X. Study on the structure and mutagenicity of a new disinfection byproduct in chlorinated drinking water. Environ. Sci. Technol. 2005, 39 (19), 7499-7508.

\section{References added in the Supporting Information}

[S1]. Kronberg, L.; Holmbom, B.; Reunanen, M.; Tikkanen, L. Identification and quantification of the Ames mutagenic compound 3-chloro-4-(dichloromethyl)-5-hydroxy-2(5H)-furanone and of its geometric isomer (E)-2-chloro-3-(dichloromethyl)-4-oxobutenoic acid in chlorine-treated humic water and drinking water extracts. Environ. Sci. Technol. 1988, 22 (9), 1097-1103.

[S2]. Richardson, S. D. Disinfection by-products: Formation and occurrence in drinking water. Encyclopedia of Environmental Health 2011, 2 (718), 110-136.

[S3]. Zhao, Y. L.; Qin, F.; Boyd, J. M.; Anichina, J.; Li, X. F. Characterization and determination of chloro- and bromo-benzoquinones as new chlorination disinfection byproducts in drinking water Anal. Chem. 2010, 82 (11), 4599-4605.

[S4]. Zhao, Y. L.; Anichina, J.; Lu, X. F.; Bull, R. J.; Krasner, S. W.; Hrudey, S. E.; Li, X. F. Occurrence and formation of chloro- and bromo-benzoquinones during drinking water disinfection. Water Res. 2012, 46 (14), 4351-4360.

[S5]. Wang, W.; Qian, Y. C.; Li, J. H.; Moe, B.; Huang, R. F.; Zhang, H. Q.; Hrudey, S. E.; Li, X. F. Analytical and toxicity characterization of halo-hydroxyl-benzoquinones as stable halobenzoquinone disinfection byproducts in treated water. Anal. Chem. 2014, 86 (10), 4982-4988. [S6]. Richardson, S. D.; Thruston, A. D.; Caughran, T. V.; Chen, P. H.; Collette, T. W.; Floyd, T. L.; Schenck, K. M.; Lykins, B. W.; Sun, G. R.; Majetich, G. Identification of new drinking water disinfection byproducts formed in the presence of bromide. Environ. Sci. Technol. 1999, 33 (19), 3378-3383.

[S7]. Richardson, S. D.; Thruston, A. D.; Rav-Acha, C.; Groisman, L.; Popilevsky, I.; Juraev, O.; Glezer, V.; McKague, A. B.; Plewa, M. J.; Wagner, E. D. Tribromopyrrole, brominated acids, and other disinfection byproducts produced by disinfection of drinking water rich in bromide. Environ. Sci. Technol. 2003, 37 (17), 3782-93.

[S8]. Pressman, J. G.; Richardson, S. D.; Speth, T. F.; Miltner, R. J.; Narotsky, M. G.; Hunter, E. S.; Rice, G. E.; Teuschler, L. K.; McDonald, A.; Parvez, S.; Krasner, S. W.; Weinberg, H. S.; McKague, A. B.; Parrett, C. J.; Bodin, N.; Chinn, R.; Lee, C. F. T.; Simmons, J. E. Concentration, chlorination, and chemical analysis of drinking water for disinfection byproduct mixtures health effects research: U.S. EPA's Four Lab Study. Environ. Sci. Technol. 2010, 44 (19), 7184-7192.

[S9]. De Leer, E. W. B.; Damste, J. S. S.; Erkeiens, C.; De Galan, L. Identification of intermediates leading to chloroform and C-4 diacids in chlorination of humic acid. Environ. Sci. Technol. 1985, 19 (6), 512-522. 
Table S8. Molecular structures of known DBPs mentioned in main text.

\begin{tabular}{|l|l|l|}
\hline Name & Formulas & Structure \\
\hline 3-ketobutanoic acid & $\mathrm{C} 4 \mathrm{H} 6 \mathrm{O} 3$ & \\
\hline 3-methyl-2-ketobutanoic acid & $\mathrm{C} 5 \mathrm{H} 8 \mathrm{O} 3$ & \\
\hline $\begin{array}{l}\text { 9-oxononanoic acid } \\
\text { oxobutenoic acid }\end{array}$ & $\mathrm{C} 9 \mathrm{H} 16 \mathrm{O} 3$ & \\
\hline
\end{tabular}


Figure S1. Optimization of derivatization conditions for carbonyl compounds in the SRFA reference standard, including temperature (a), amounts of GRP reagent (b), incubation time (c) and acidity (d).
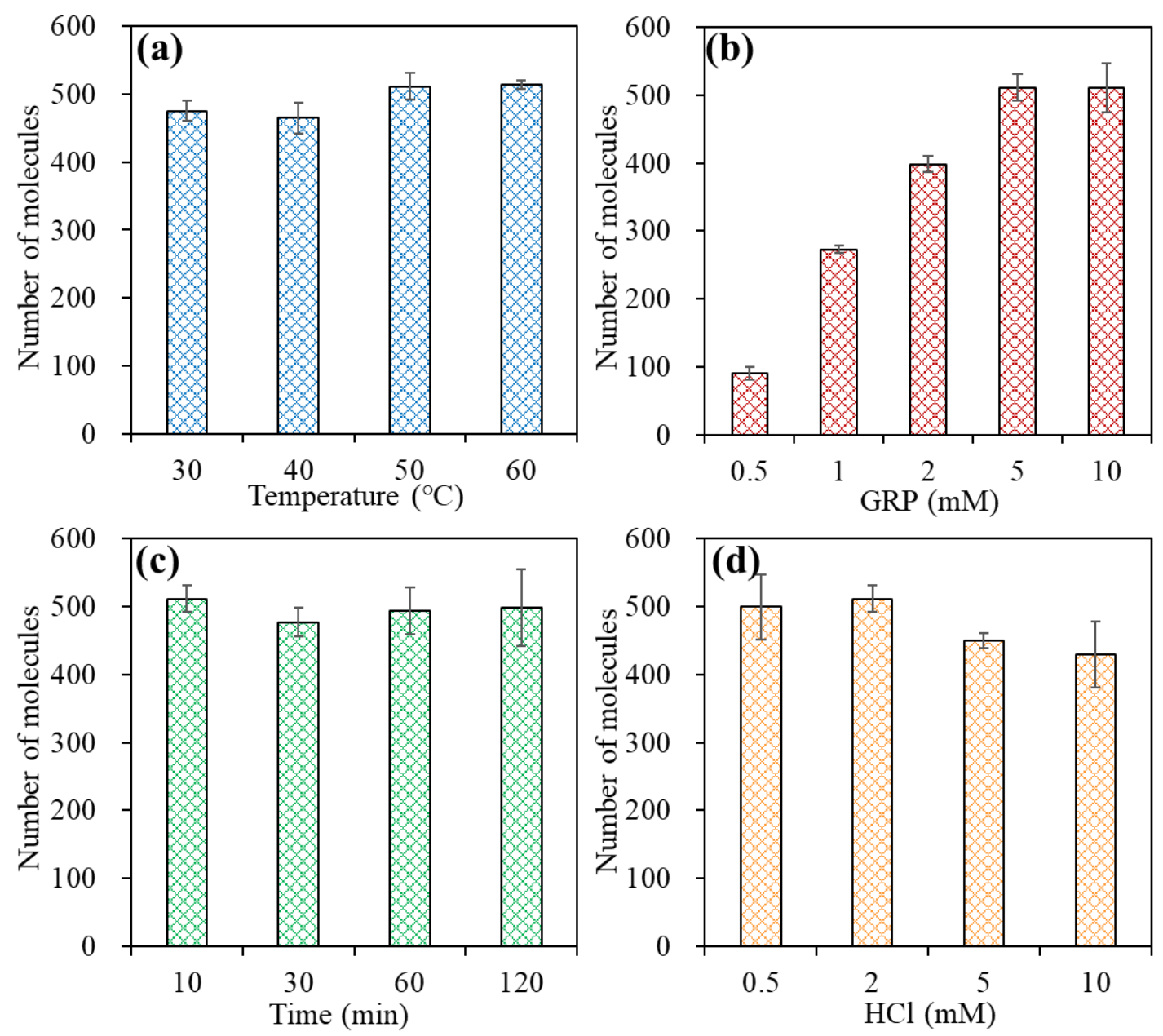
Figure S2. Venn diagram on number of carbonyl compounds identified in the three parallel SRFA aqueous samples.

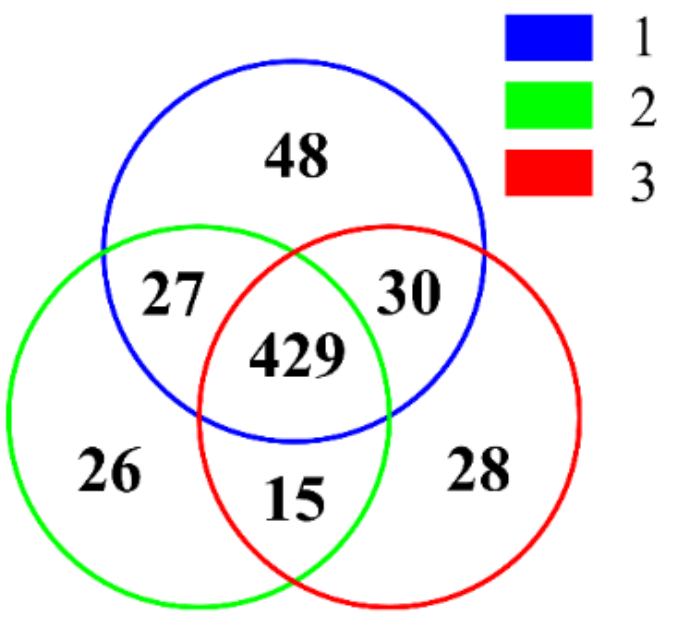


Figure S3. An illustration on derivatization reaction of a representative carbonyl compound $\left(\mathrm{C}_{10} \mathrm{H}_{10} \mathrm{O}_{6}\right)$ in the $\mathrm{SRFA}$ reference standard with $\mathrm{D}_{0} / \mathrm{D}_{5}$ GRP (a), and $\mathrm{MS}^{2}$ spectra of the corresponding $\mathrm{D}_{0} / \mathrm{D}_{5}$-GRP derivatives $\left(\mathrm{b},\left[\mathrm{C}_{17} \mathrm{H}_{18} \mathrm{~N}_{3} \mathrm{O}_{6}\right]^{+}\right.$and $\left.\left[\mathrm{C}_{17} \mathrm{H}_{13} \mathrm{D}_{5} \mathrm{~N}_{3} \mathrm{O}_{6}\right]^{+}\right)$.

(a)

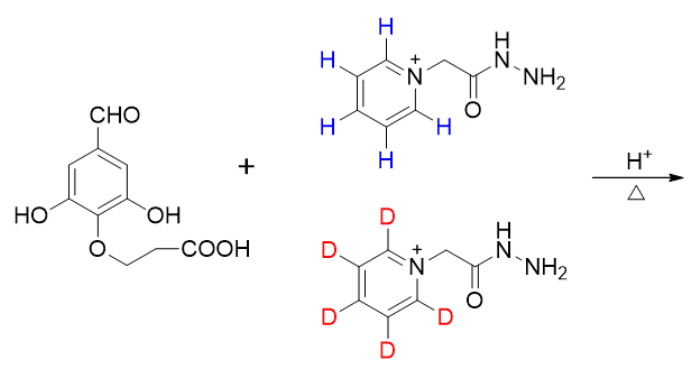

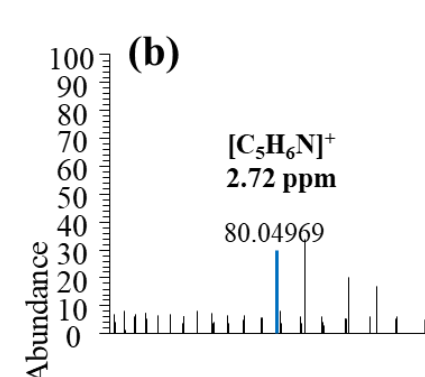

$\left[\mathrm{C}_{7} \mathrm{H}_{6} \mathrm{NO}^{+}\right.$

$\underset{1.43 \mathbf{p p m}}{\left[\mathrm{C}_{7} \mathbf{H}_{6} \mathbf{N O}^{+}\right.}$

$\underset{120.04456}{\left[\mathrm{C}_{7} \mathbf{H}_{6} \mathbf{N O}^{+}\right.}$

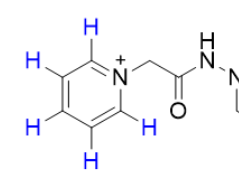

|
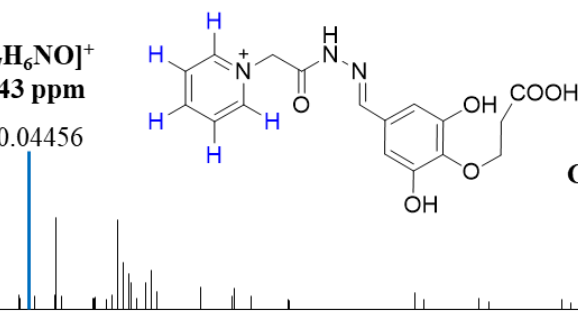

\section{[M-}
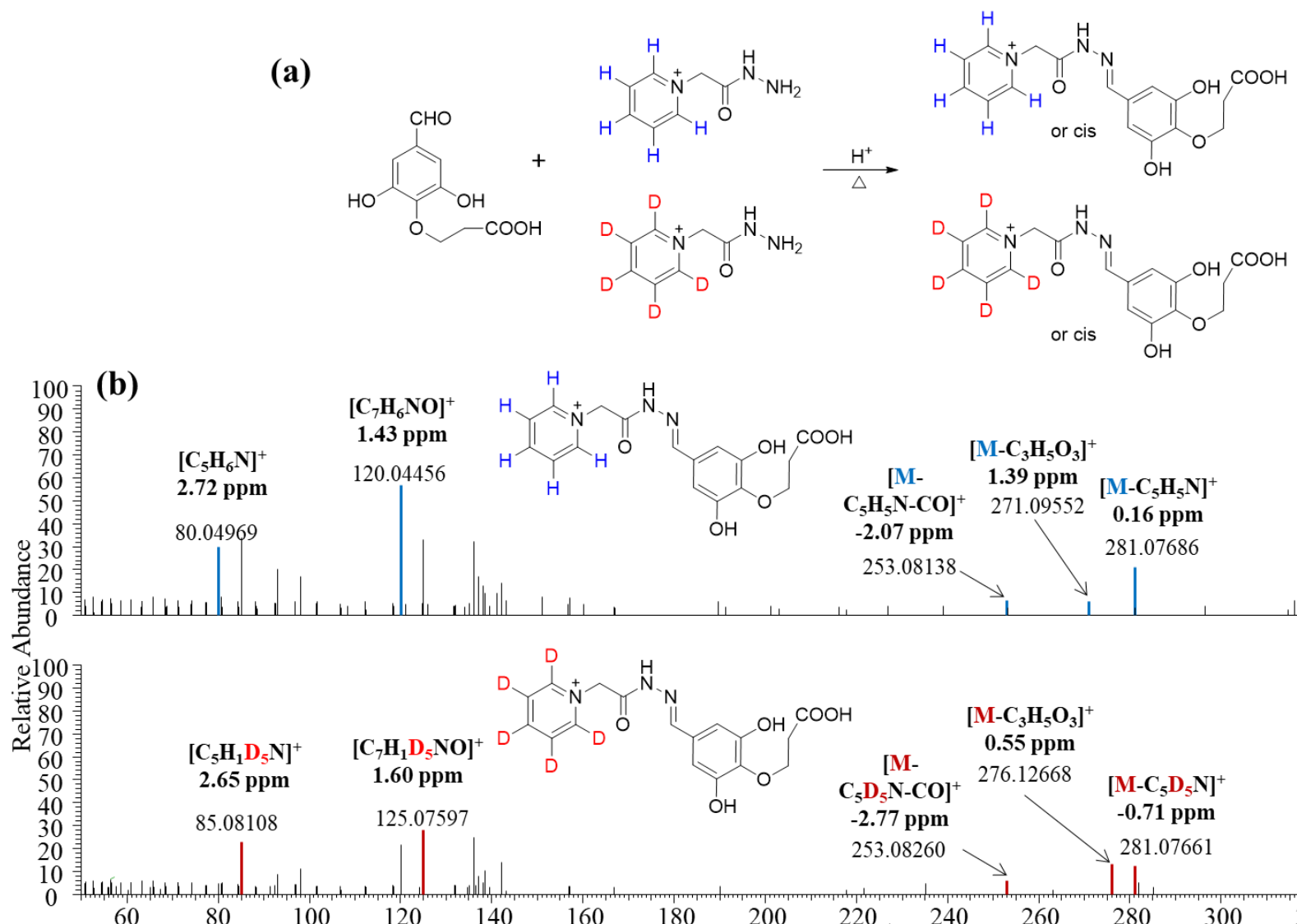

$\mathrm{C}_{5} \mathrm{H}_{5} \mathrm{~N}-\mathrm{CO}$

$-2.07 \mathrm{ppm}$

$\left[\mathrm{M}-\mathrm{C}_{3} \mathrm{H}_{5} \mathrm{O}_{3}\right]^{+}$

$1.39 \mathrm{ppm}\left[\mathrm{M}-\mathrm{C}_{5} \mathrm{H}_{5} \mathrm{~N}\right]^{+}$

342.10773
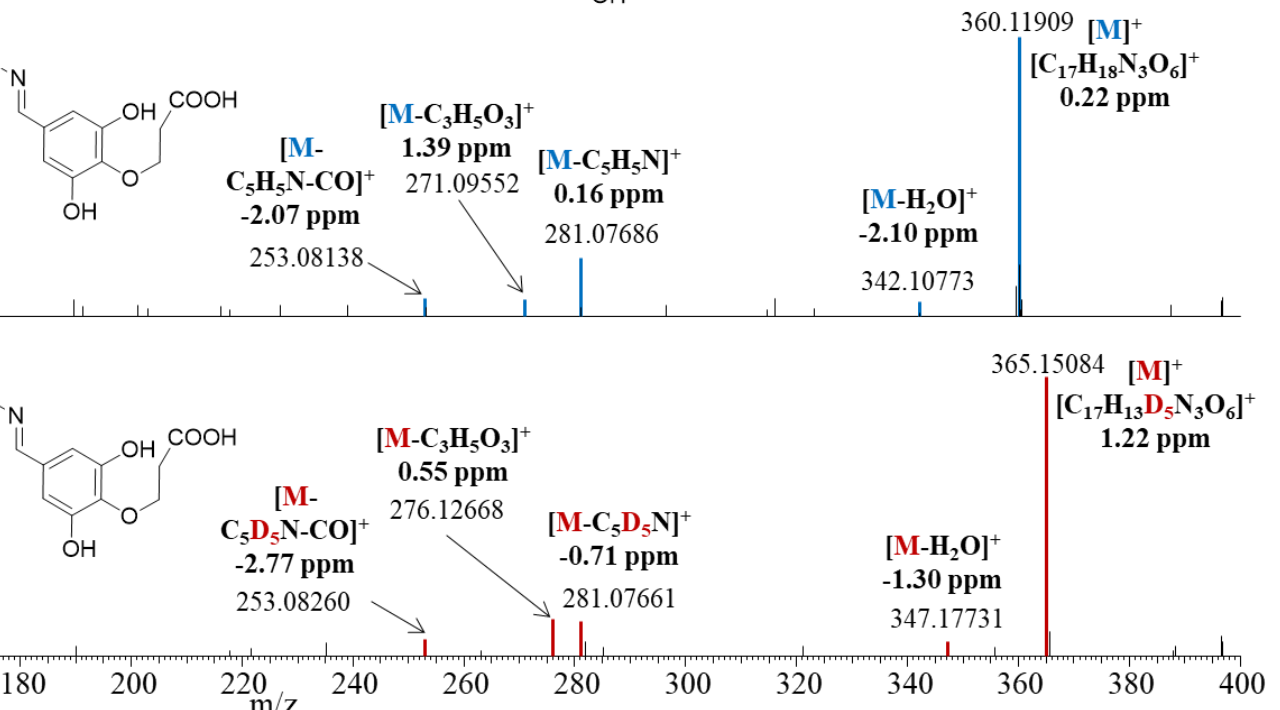
Figure S4. Proposed average structural model of the SRFA reference standard (a), proposed structure of a lignin molecule $\left(\mathrm{C}_{24} \mathrm{H}_{28} \mathrm{O}_{11}, \mathrm{~b}\right)$, and several depolymerization products of lignin (c). The moieties of hydroxyphenyl alkyl ketones were circled by red dotted line in the structures of fulvic acid and lignin.

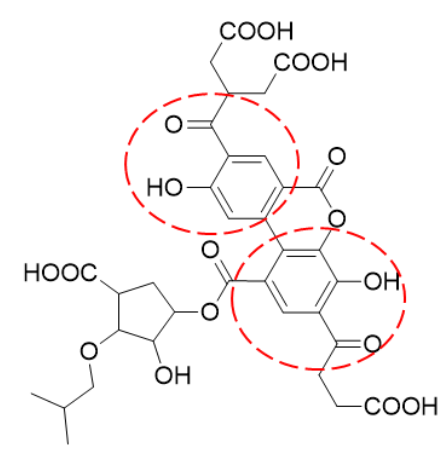

(a) fulvic acid

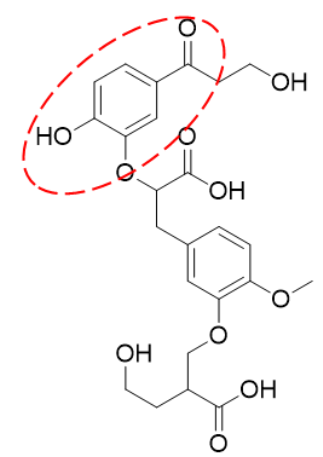

(b) lignin

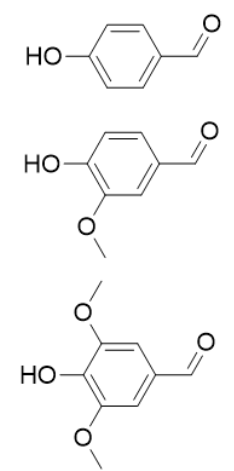

(c) depolymerization products of lignin 
Figure S5. Relative abundance and formation of carbonyl molecules in the SRFA solution after ozonation (a,d), chlorination (b,e), and chloramination $(\mathrm{c}, \mathrm{f})$ by van Krevelen plots. In d-f, red points referred to the newly produced carbonyl compounds (DBPs), and black triangles were original carbonyl compounds existed and remained in the solution.
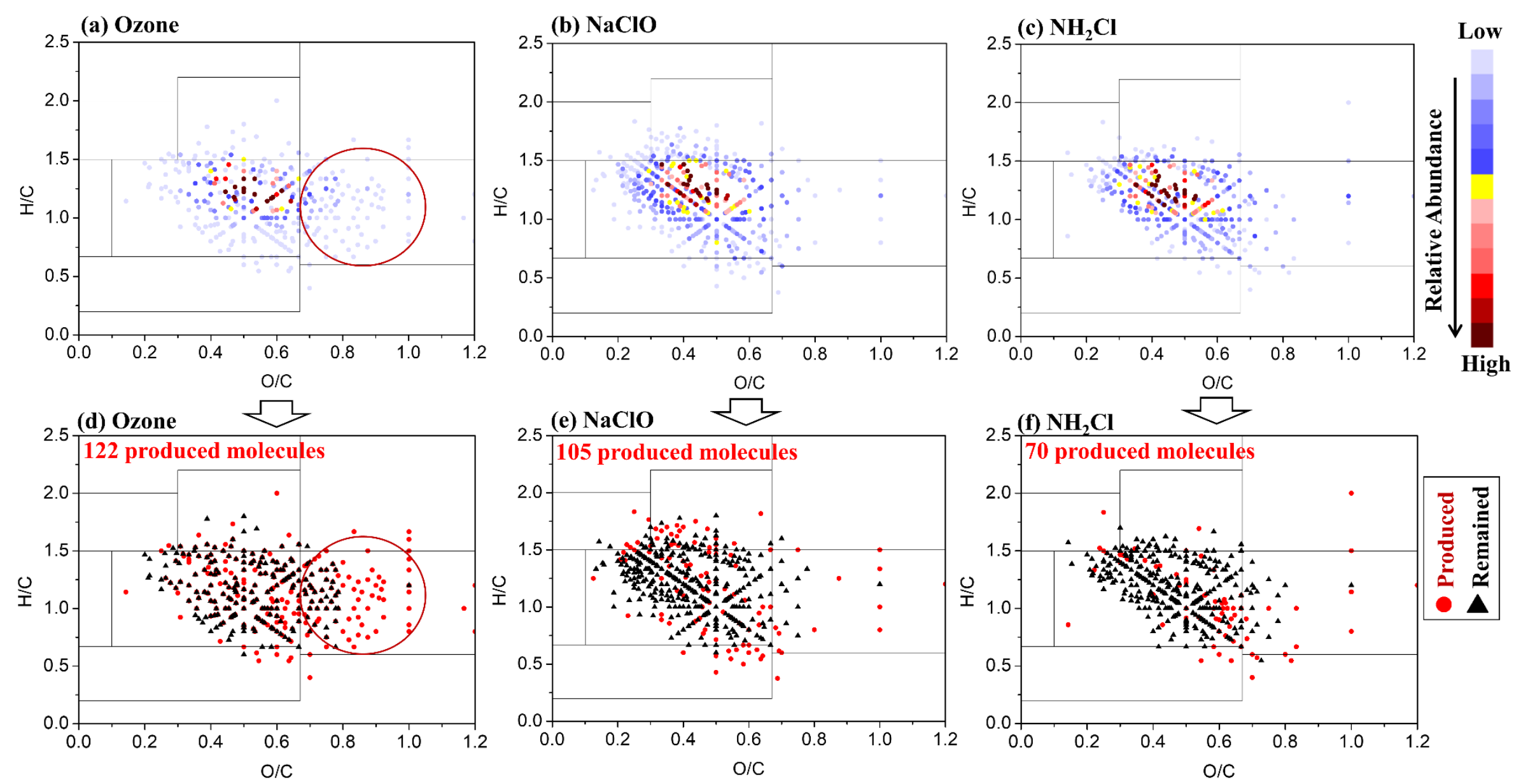
Figure S6. Molecular characteristic alteration of carbonyl compounds in HOCs region $(0.67 \leqslant \mathrm{O} / \mathrm{C} \leqslant 1.2$ and $0.6<\mathrm{H} / \mathrm{C} \leqslant 1.5)$ after ozonation. The number of produced (red), remained (black) and disappeared (green) carbonyl compounds shown by oxygen number (a); DBE composition of the produced carbonyl compounds (carbonyl DBPs) in $\mathrm{O}_{4-10}$ species (b); KMD plots of representative carbonyl DBPs with proposed structures (c).
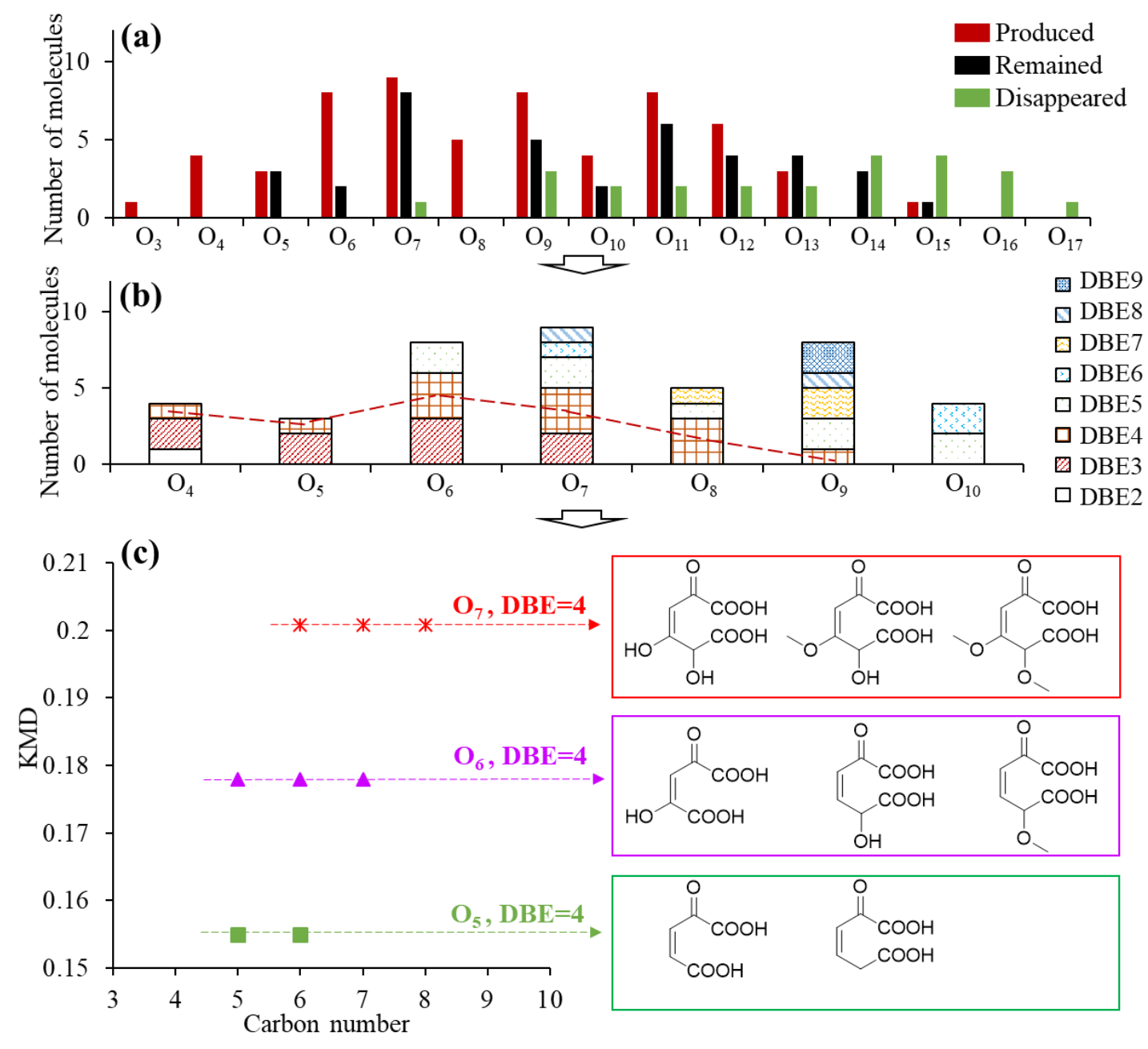
Figure S7. Distribution of carbonyl compound numbers shown by oxygen number, and DBE distribution shown by oxygen number after ozonation (a, b), chlorination (c, d) and chloramination (e, f).
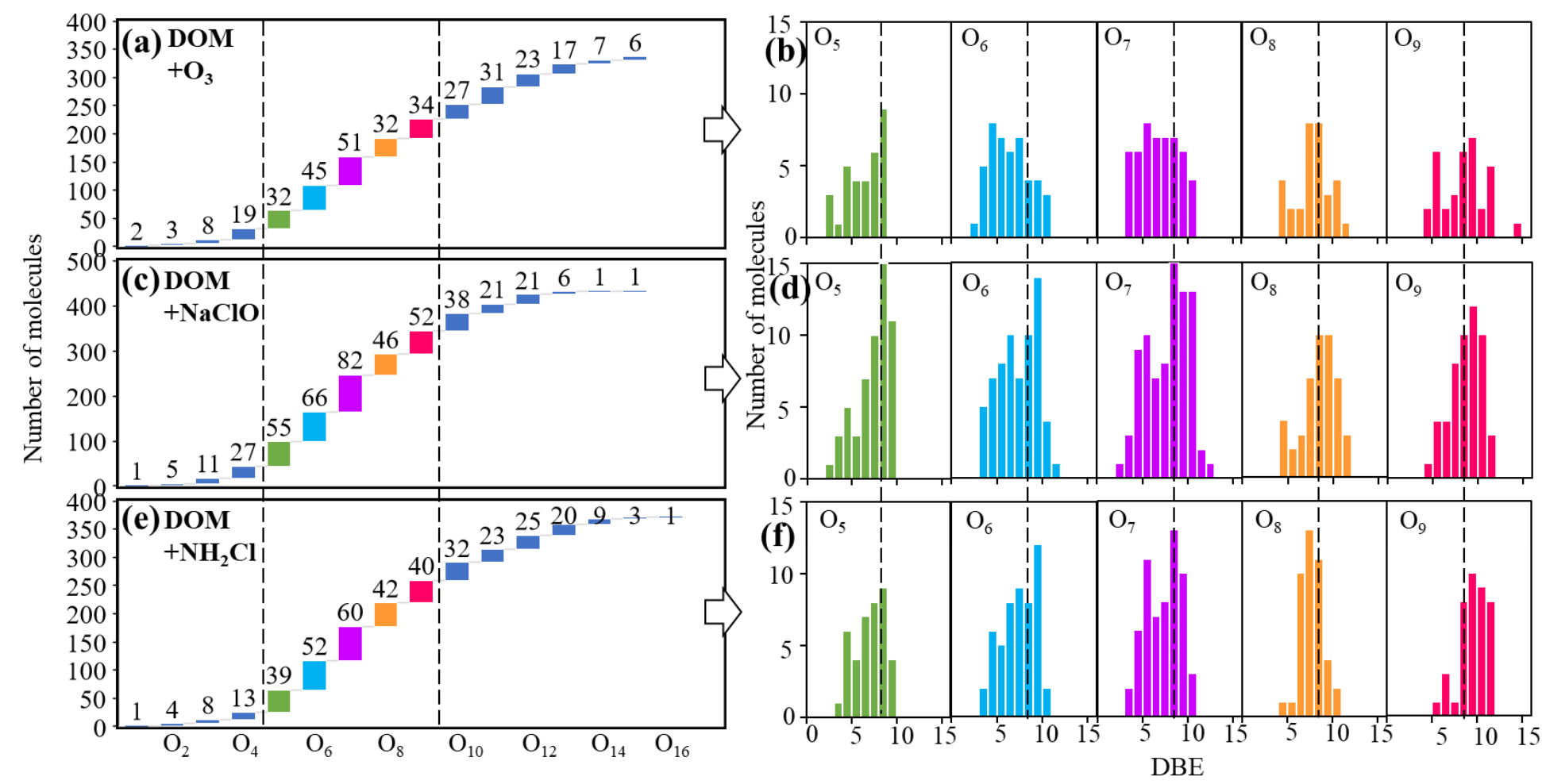
Figure S8. KMD plots of Cl-carbonyl-DBP homologous $\left(\mathrm{O}_{3}, \mathrm{DBE}=2-3 ; \mathrm{O}_{4}, \mathrm{DBE}=\right.$ $4 ; \mathrm{O}_{5}, \mathrm{DBE}=5$ ) in chloramine-treated SRFA solution.

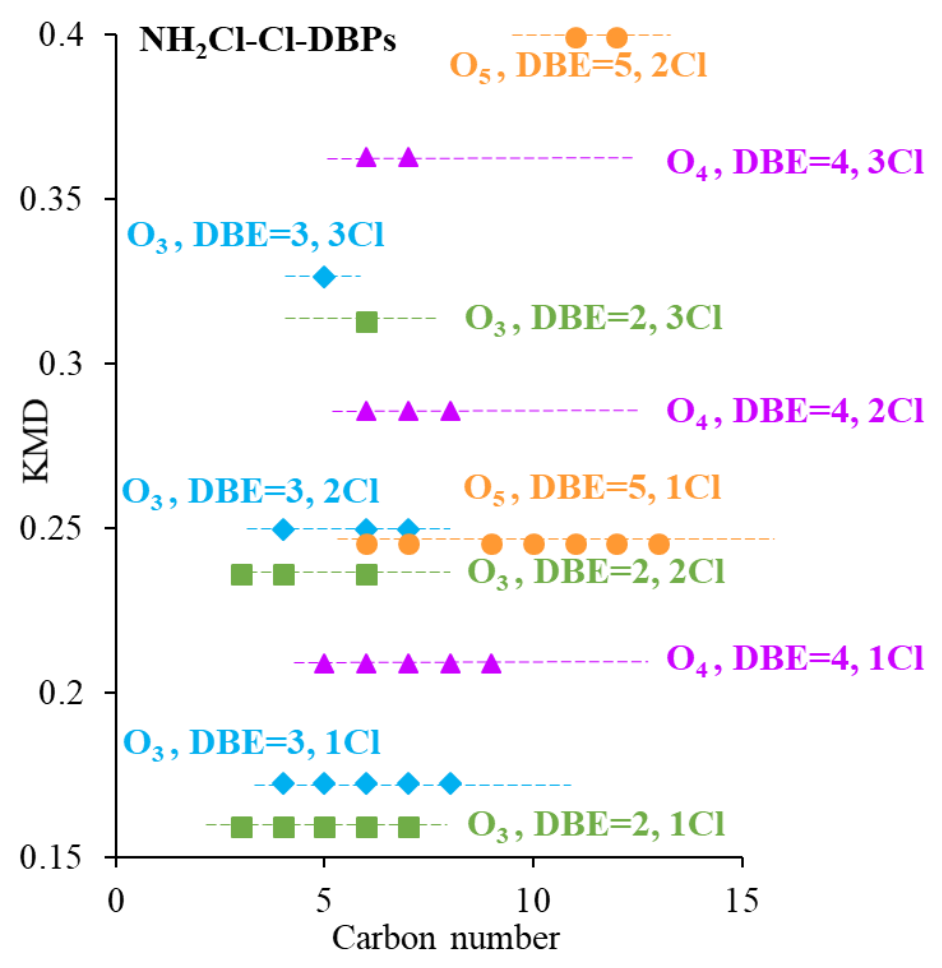


Figure S9. $\mathrm{MS}^{2}$ spectra of $\mathrm{D}_{0} / \mathrm{D}_{5}-\mathrm{GRP}$ derivatives of $\mathrm{C}_{7} \mathrm{H}_{5} \mathrm{O}_{5} \mathrm{Cl}$ (a) and $\mathrm{C}_{9} \mathrm{H}_{9} \mathrm{O}_{5} \mathrm{Cl}$ (b) with $\mathrm{DBE}=5$ in $\mathrm{O}_{5}$ species.
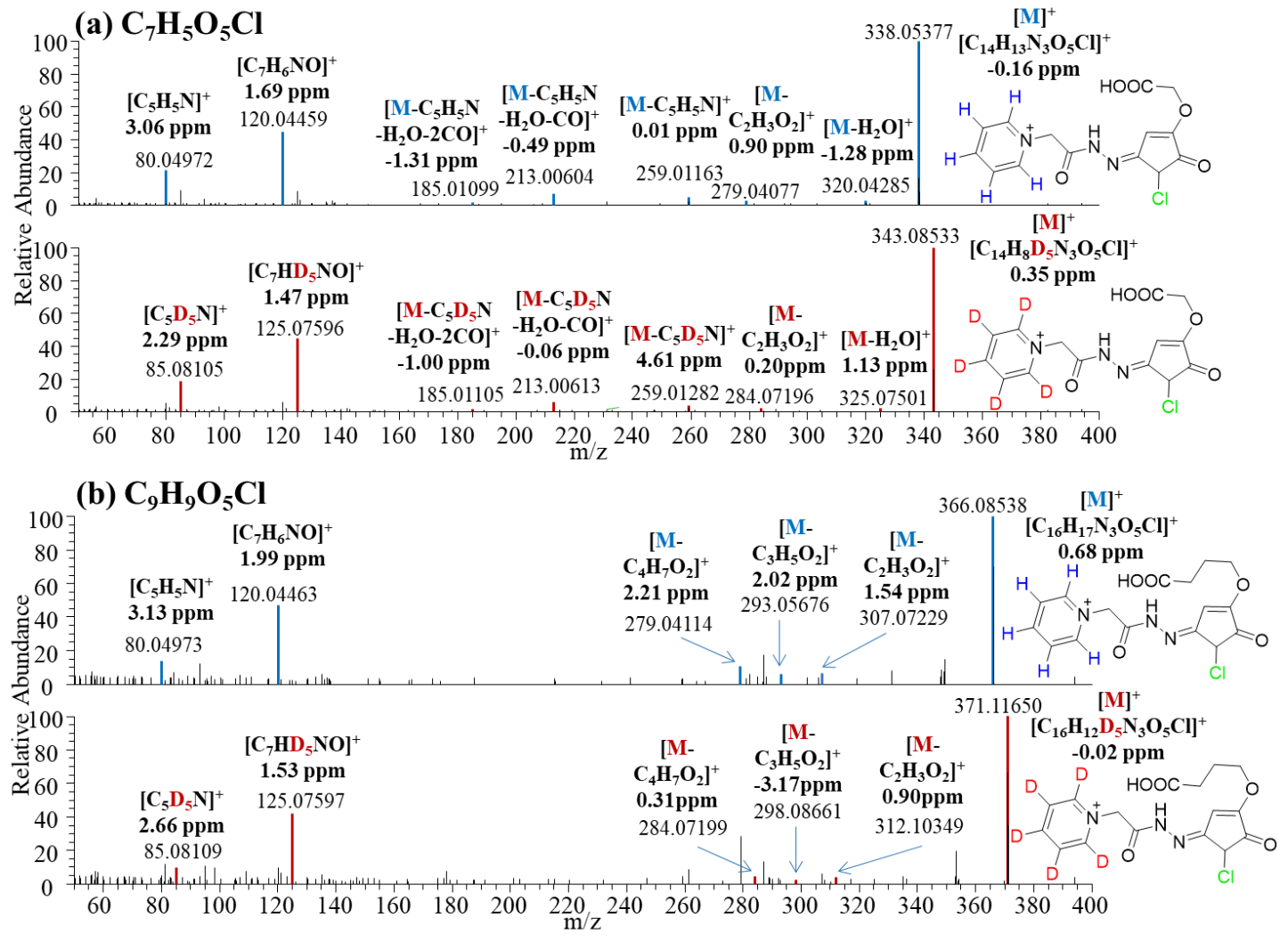
Figure S10. $\mathrm{MS}^{2}$ spectra of $\mathrm{D}_{0} / \mathrm{D}_{5}$-GRP derivatives of $\mathrm{C}_{6} \mathrm{H}_{4} \mathrm{O}_{4} \mathrm{Cl}_{2}\left(\mathrm{a}, \mathrm{DBE}=4\right.$ in $\mathrm{O}_{4}$ species), $\mathrm{C}_{5} \mathrm{H}_{5} \mathrm{O}_{3} \mathrm{Cl}$ (b, DBE $=3$ in $\mathrm{O}_{3}$ species), $\mathrm{C}_{5} \mathrm{H}_{6} \mathrm{O}_{3} \mathrm{Cl}_{2}$ (c, $\mathrm{DBE}=2$ in $\mathrm{O}_{3}$ species), and $\mathrm{C}_{4} \mathrm{H}_{3} \mathrm{O}_{3} \mathrm{Cl}$ (d, $\mathrm{DBE}=3$ in $\mathrm{O}_{3}$ species).

(a) $\mathrm{C}_{6} \mathrm{H}_{4} \mathrm{O}_{4} \mathrm{Cl}_{2}$

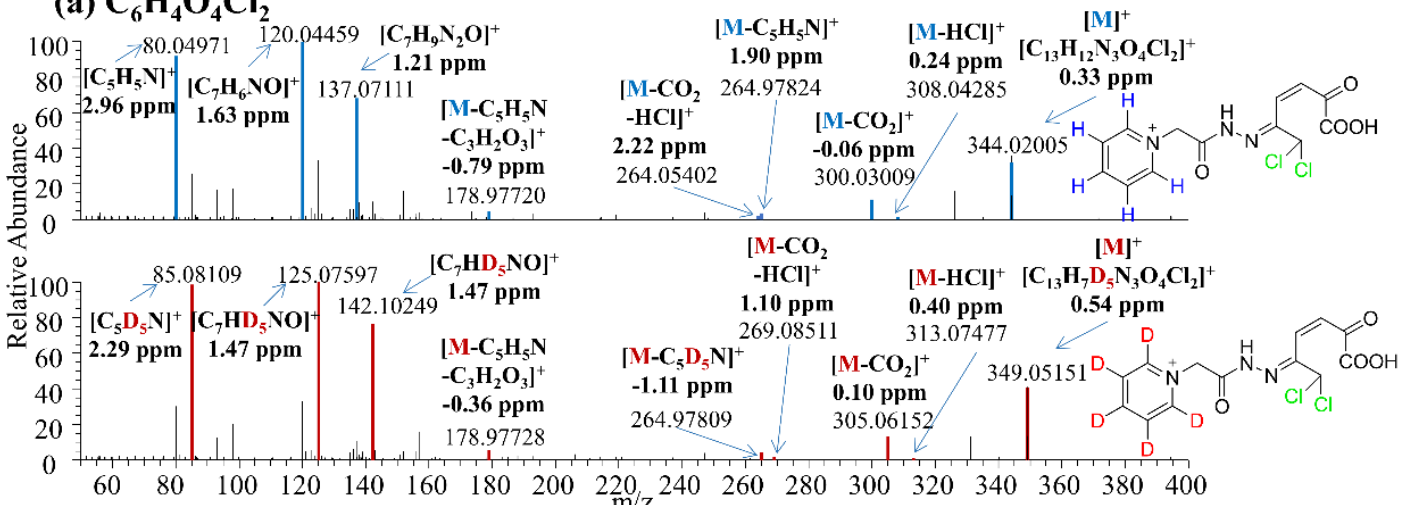

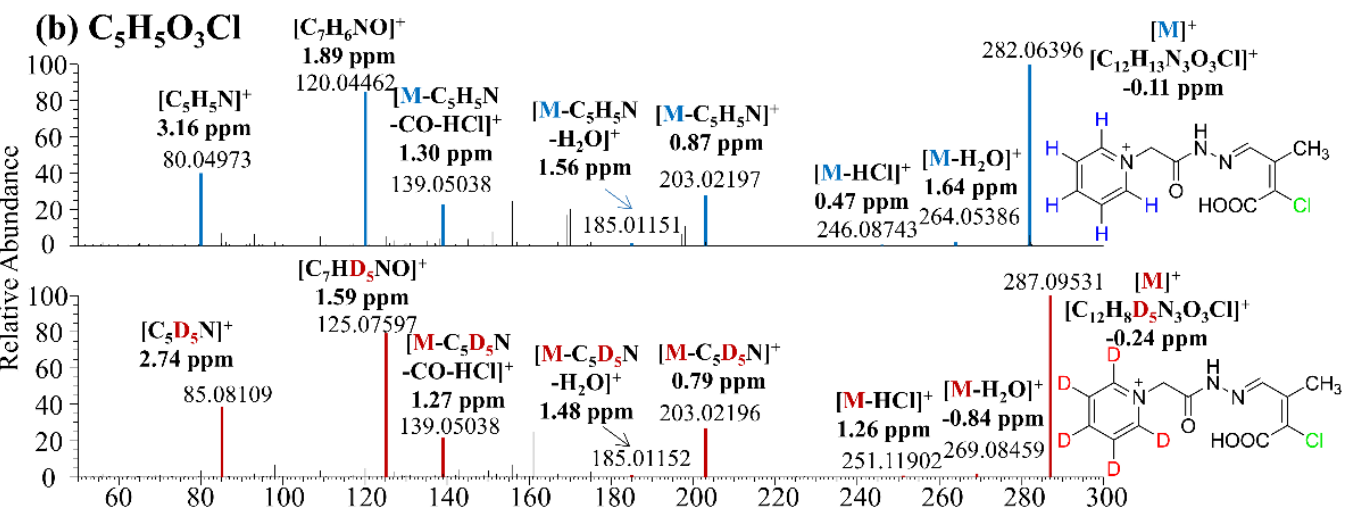

\section{(c) $\mathrm{C}_{5} \mathrm{H}_{60} \mathbf{O}_{3} \mathbf{C l}_{2}$}
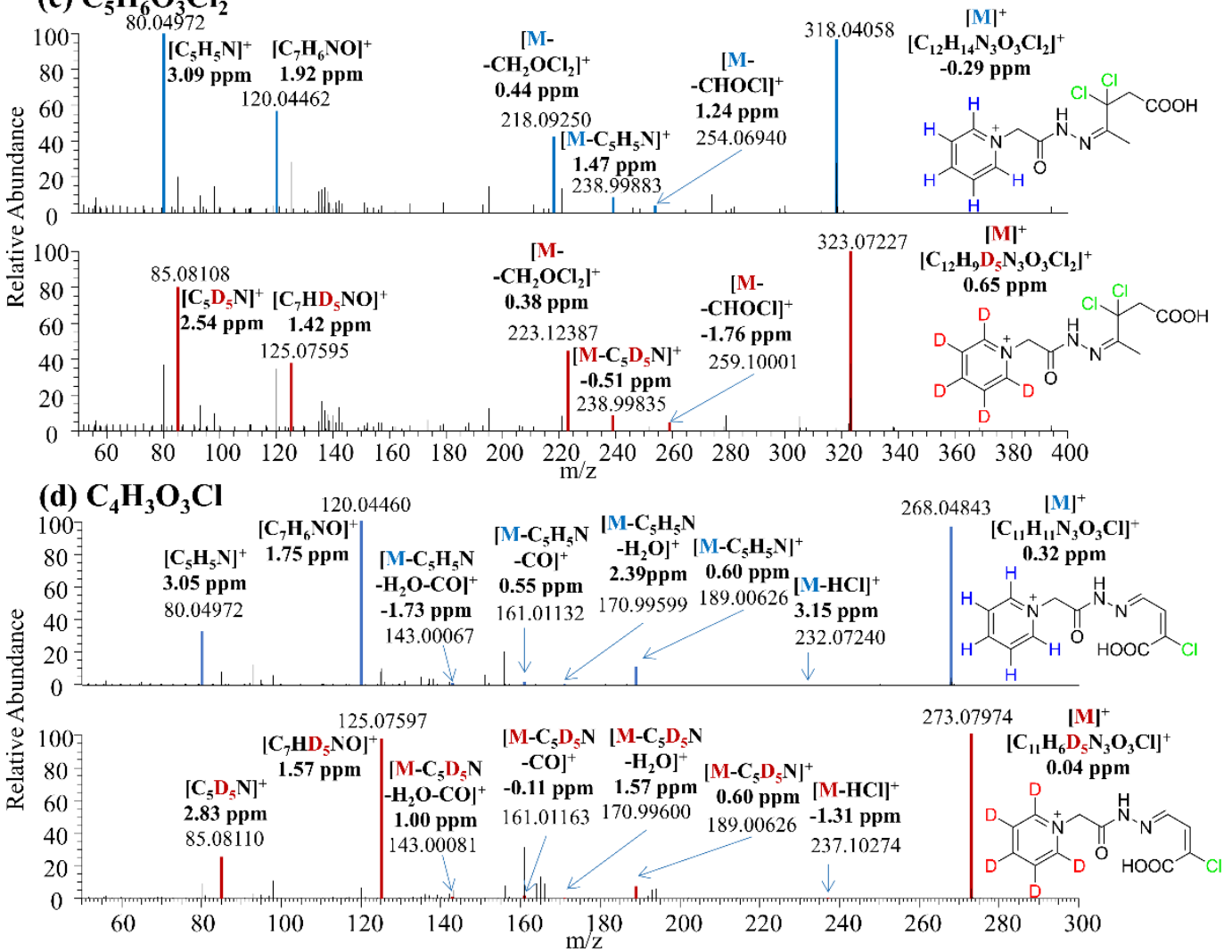
Figure S11. Molecular characteristics of Br-carbonyl-DBPs after chlorination and chloramination. The number of Br-carbonyl-DBPs induced by chlorine (green) and chloramine (blue) shown by oxygen number (a); DBE composition of $\mathrm{O}_{3}$ species (b); KMD plots of the Br-carbonyl-DBP homologous $\left(\mathrm{O}_{3}, \mathrm{DBE}=3\right)$ with representative proposed structures (c); $\mathrm{MS}^{2}$ spectra of $\mathrm{D}_{0} / \mathrm{D}_{5}$-GRP derivatives of $\mathrm{C}_{4} \mathrm{H}_{3} \mathrm{O}_{3} \mathrm{Br}$ (d).
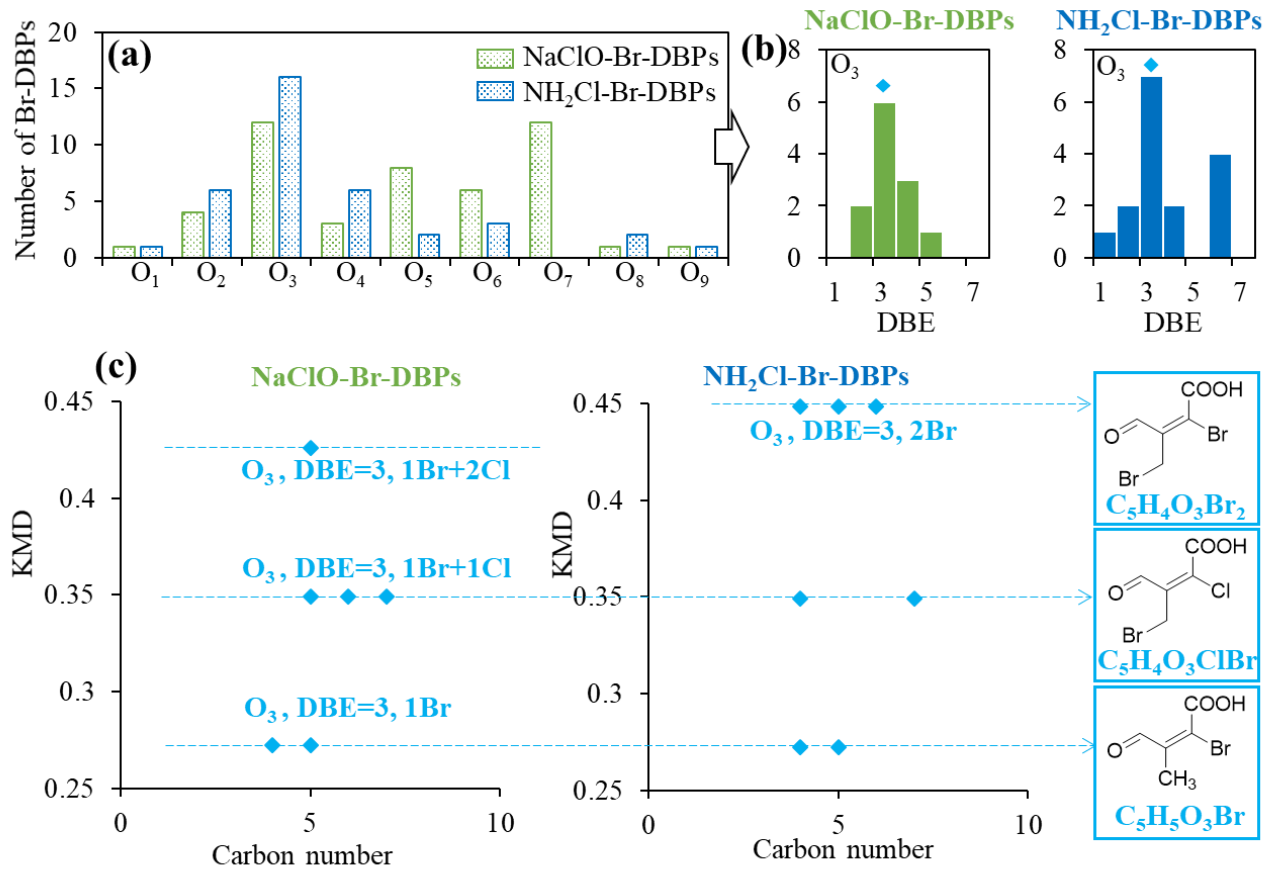

(d)

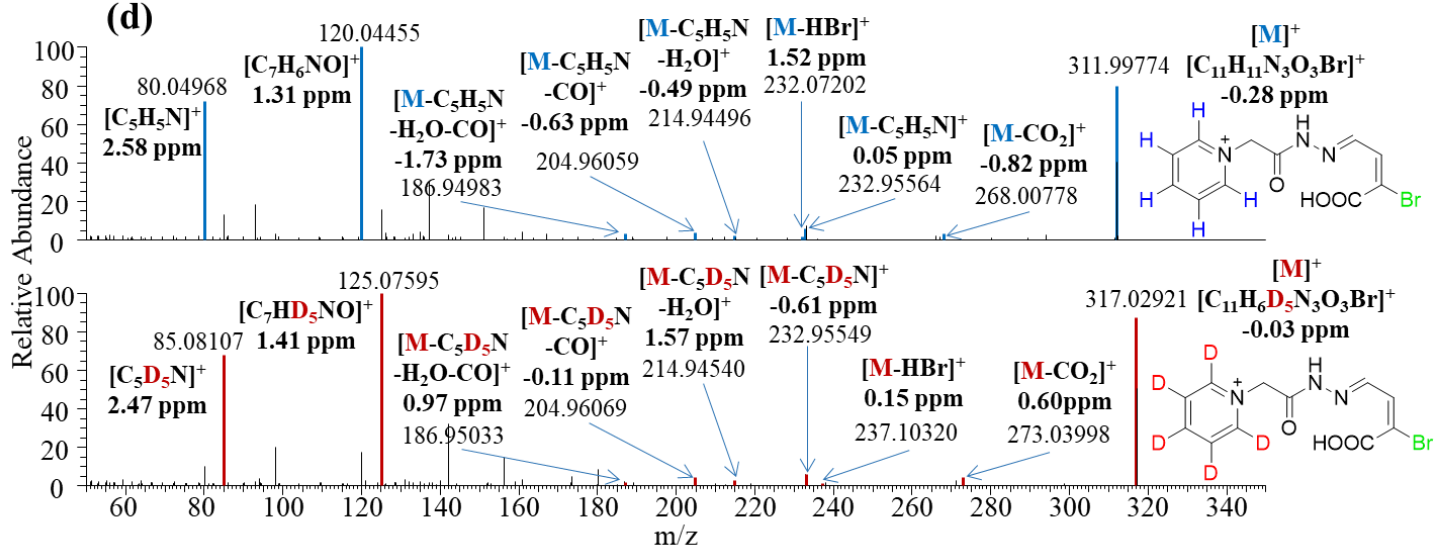


Figure S12. Molecular characteristics of I-carbonyl-DBPs after chloramination and ozonation. The number of I-carbonyl-DBPs induced by chloramine (blue) and ozone (red) shown by oxygen number (a); DBE composition of $\mathrm{O}_{3-5}$ class species (b); KMD plots of the I-carbonyl-DBP homologous $\left(\mathrm{O}_{3}, \mathrm{DBE}=2-3 ; \mathrm{O}_{4}, \mathrm{DBE}=4 ; \mathrm{O}_{5}, \mathrm{DBE}=\right.$ 5) with representative proposed structures (c); $\mathrm{MS}^{2}$ spectra of $\mathrm{D}_{0} / \mathrm{D}_{5}$-GRP derivatives of $\mathrm{C}_{4} \mathrm{H}_{3} \mathrm{O}_{3} \mathrm{I}(\mathrm{d})$.
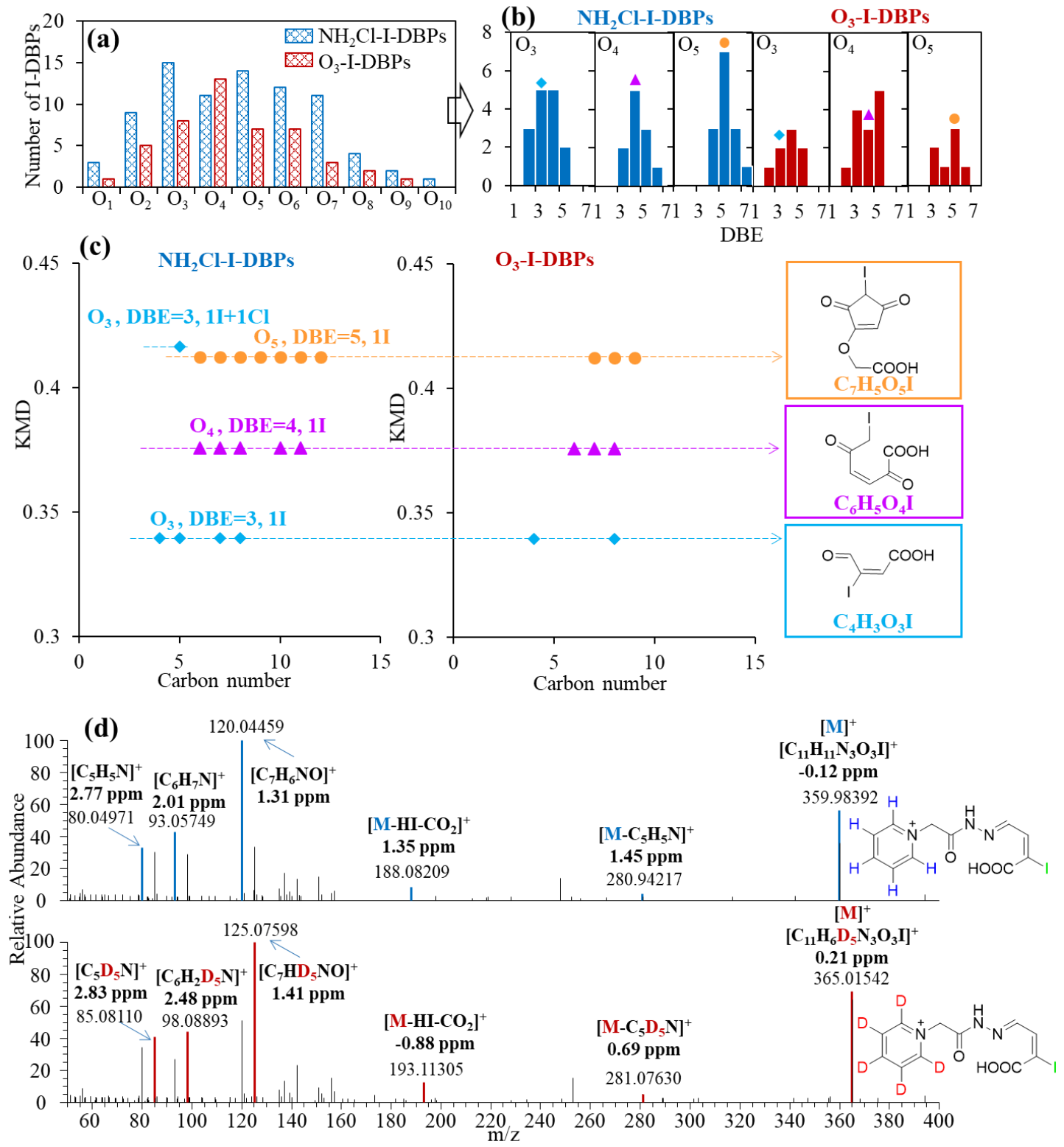